\title{
Stomach and colonic microbiome of wild Japanese macaques
}

\section{$\operatorname{AUTHOR}(S)$ :}

Lee, Wanyi; Hayakawa, Takashi; Kurihara, Yosuke; Hanzawa, Maho; Sawada, Akiko; Kaneko, Akihisa; Morimitsu, Yoshiki; ... Ito, Tsuyoshi; Honda, Takeaki; Hanya, Goro

\section{CITATION:}

Lee, Wanyi ...[et all. Stomach and colonic microbiome of wild Japanese macaques. American Journal of Primatology 2021, 83(5): e23242.

\section{ISSUE DATE:}

2021-05

\section{URL:}

http://hdl.handle.net/2433/267535

\section{RIGHT:}

This is the peer reviewed version of the following article: [Lee, W., Hayakawa, T., Kurihara, Y., Hanzawa, M., Sawada, A., Kaneko, A., Morimitsu, Y., Natsume, T., Aisu, S., Ito, T., Honda, T., \& Hanya, G. Stomach and colonic microbiome of wild Japanese macaques. Am J Primatol. 2021; 83:e23242], which has been published in final form at

https://doi.org/10.1002/ajp.23242. This article may be used for non-commercial purposes in accordance with Wiley Terms and Conditions for Use of Self-Archived Versions. This article may not be enhanced, en riched or otherwise transformed into a derivative work, without express permission from Wiley or by statutory rights under applicable legislation. Copyright notices must not be removed, obscured or modified. The article must be linked to Wiley's version of record on Wiley Online Library and any embedding, framing or otherwise making available the art ... 


\section{Stomach and colonic microbiome of wild Japanese macaques}

2 Short title: Gut-site-specific microbiome

3 Wanyi Lee ${ }^{1 *}$, Takashi Hayakawa ${ }^{2,3}$, Yosuke Kurihara ${ }^{1,4}$, Maho Hanzawa ${ }^{5}$, Akiko Sawada ${ }^{1,6}$,

4 Akihisa Kaneko $^{1}$, Yoshiki Morimitsu ${ }^{7}$, Takayoshi Natsume ${ }^{1}$, Seitaro Aisu ${ }^{1}$, Tsuyoshi Ito ${ }^{1}$,

$5 \quad$ Takeaki Honda ${ }^{1}$, Goro Hanya ${ }^{1}$

$6 \quad{ }^{1}$ Primate Research Institute, Kyoto University, Inuyama, Aichi, Japan

$7 \quad{ }^{2}$ Faculty of Environmental Earth Science, Hokkaido University, Sapporo, Hokkaido, Japan

$8 \quad{ }^{3}$ Japan Monkey Centre, Inuyama, Aichi, Japan

$9 \quad{ }^{4}$ Center for Education and Research in Field Sciences, Faculty of Agriculture, Shizuoka

10 University, Hamamatsu, Japan

$11{ }^{5}$ Department of Zoology, Graduate School of Science, Kyoto University, Kyoto, Japan

$12{ }^{6}$ Academy of Emerging Sciences, Chubu University, Kasugai, Aichi, Japan

$13{ }^{7}$ Institute of Natural and Environmental Sciences, University of Hyogo, Sanda, Hyogo, Japan

$14 *$ To whom correspondence should be addressed; Correspondence to

15 wanyi23333@gmail.com

16 


\section{Highlights}

- Diversity, composition and function vary between stomach and colonic microbiome of Japanese macaques

- Stomach microbiome is more enriched in microbes that metabolize simple sugar, whereas the colonic microbiome has more fiber-degrading microbes.

\section{Abstract}

Within the gastrointestinal tract, the physiochemical microenvironments are highly diversified among the different stages of food digestion. Accordingly, gut microbiome composition and function vary at different gut sites. In this study, we examine and compare the compositional and functional potential between the stomach and colonic microbiome of wild Japanese macaques (Macaca fuscata yakui) living in the evergreen forest of Yakushima Island.

We find a significantly lower microbial diversity in the stomach than in the colon, possibly due to the stomach's acidic and aerobic environment, which is suboptimal for microbial survival. According to past studies, the microbial taxa enriched in the stomach are aero- and acid-tolerant. By functional prediction through PICRUSt2, we reveal that the stomach microbiome is more enriched in pathways relating to the metabolism of simple sugars. On the contrary, the colonic microbiota is more enriched with fiber-degrading microbes, such as those from Lachnospiracea, Ruminococcaceae and Prevotella. Our study shows a clear difference in the microbiome between the stomach and colon of Japanese macaques in both composition and function. This study provides a preliminary look at the alpha diversity and taxonomic composition within the stomach microbiome of Japanese macaques, a hindgut-fermenting non-human primate.

Keywords: primates, gut microbiome, Japanese macaques, gut sites

\section{Introduction}

Along the gastrointestinal (GI) tract, the microbiome typically diversifies in relation to the digestive functions (mechanical, chemical, and microbial breakdown) and corresponding 

physiochemical environment at different gut sites (Gu et al., 2013; Hillman, Lu, Yao, \& community in the upper GI tract is likely suited to the breakdown of simple sugars and proteins, while the microbiome in the lower GI tract is likely suited to complex plant polysaccharides. In addition, how different microbes adapt to the physiochemical environment at different gut sites may determine the acquisition/colonization mechanism of the gut microbiome (Merrell, Goodrich, Otto, Tompkins, \& Falkow, 2003; Seedorf et al., 2014; Vega, 2019).

Many studies on the gut microbiome-host relationship have focused on the colonic microbiome, which plays a major role in fermentation. In the anaerobic environment of the colon, gut microbes carry out fermentation to transform food materials into short-chain fatty acid and other nutrients, serving as energy and nutritional source for the hosts. It is estimated that the colon alone contains over $70 \%$ of the bacteria residing in the body (in the case of humans (Jandhyala et al., 2015)). Compared to the other GI sites, which usually require invasive sampling, it is possible to study the colonic microbiome non-invasively using fecal samples. Therefore, despite the potential differences among GI sites, the gut microbiome studies have mainly focused on the microbial community in the colon/hindgut of animals (Clayton et al., 2019).

Compared with the colon, the stomach, which carries out chemical digestion, presents a different environment for most bacteria, including its low-pH environment and short transit time. In past studies on humans and animals (captive rats, swine, mice, baboons and redshanked doucs), bacterial diversity in the upper sections of the GI tract, such as the stomach, tends to be lower than that in the lower sections, such as the colon (Clayton et al., 2019; Stevens \& Hume, 1998). Moreover, the function of microbes in the stomach is potentially different from that in the colon. For example, pathways related to environmental information processing 
increases in the upper GI tract of house mice, suggesting an active material exchange between gut microbes and the digestive organ (D. Li et al., 2019).

Despite the environmental differences between the stomach and colon, there have been few studies devoted to the stomach microbiome. An understanding of the stomach microbiome is, however, important in providing insights into how the animals acquire gut microbes and how the microbes distribute to the lower GI tract. Mammals are generally born with a sterile GI, and thus they acquire gut microbes from the environment. Even after acquisition, microbes vary in their ability to establish a population under various physiochemical environments across the GI tract. While some studies have pointed out the difference in microbiome between the stomach/foregut and colon/hindgut of the animals, the study subjects have only been a few species of nonhuman primates (NHPs), mostly with a focus on the captive foregut-fermenting species (e.g. red-shanked doucs (Amato et al., 2016; Clayton et al., 2019), black and white colobus monkeys, and langurs (Amato et al., 2016)). Despite the fact that most NHPs are hindgut-fermenters, there is clearly a lack of knowledge on the diversity and distribution of microbial communities within the hindgut-fermenting NHPs. Such knowledge would provide basic information regarding the gut microbiome of the hindgut-fermenting NHPs. Furthermore, comparing NHP gut microbiome of different fermentative strategies would improve our understanding of the special digestive adaptations of the foregut-fermenters and thus the evolutionary trajectory of primate feeding strategy.

In this work, we studied wild Japanese macaques (Macaca fuscata yakui) inhabiting warm-temperate evergreen forest in Yakushima Island, Japan, to understand the spatial difference in the gut microbiome between the stomach and colon. Japanese macaques feed on a considerable amount of mature leaves and other fibrous foods to survive the food-scarce seasons (Hanya, 2004; Hanya, Noma, \& Agetsuma, 2003; Hill, 1997; Kurihara, Kinoshita, Shiroishi, \& Hanya, 2020). They spend approximately 35\% of their annual feeding time on 
101 fibrous leaves and shoots (Hill, 1997). Of this, the neutral detergent fiber content of the major

102 food leaves could be as high as 42\% (Hanya, Kiyono, Takafumi, Tsujino, \& Agetsuma, 2007).

103 It is therefore critical to understand how the gut microbiome contributes to the macaques'

104 nutrition while considering the macaques' intake of fibrous food items.

105 In this study we aimed (1) to examine and compare the microbiome compositions of 106 Japanese macaques at two different gut sites, the stomach and colon, and then (2) to infer and 107 compare the functions of the gut microbiome at different gut sites. Our hypothesis is that the 108 stomach microbiome will be less diverse and related to environmental information processing 109 and simple sugar metabolism, while the colonic microbiome will be more diverse and enriched 110 with pathways involving fiber digestion. This study aims to improve our understanding of the

111 hindgut-fermenting NHPs' gut microbiome, while focusing on the filtering effect imposed by 112 different GI sites on the microbiome diversity and function.

\section{Methods}

\section{Sample collection}

116 We collected stomach content and colon samples from a total of 13 individual 117 macaques inhabiting the coastal area of Yakushima Island, Japan $\left(30^{\circ} \mathrm{N}, 131^{\circ} \mathrm{E}\right)$ : males and

118 females from each of the three troops (Umi A, Umi B and Umi C) during July 11-14, 2017, 119 May 27-30, 2018, and September 25-28, 2019 (Supplementary Table 1). We sampled each 120 monkey only once. In 2017, we only collected colonic samples: one male and one female from

121 Umi A and one female from Umi B. In 2018, we collected both stomach and colon samples 122 from one male and one female from each of the three troops. In 2019, we collected stomach and colon samples from one male and one female from Umi A and Umi C. These individuals were captured for the purpose of attaching GPS collars. One of us (A. K.), as a vet, darted the animals with VARIO 1V ® Telinject and anesthetized them with 40 or $60 \mathrm{mg}$ of ketamine, 0.2 
or $0.3 \mathrm{mg}$ of medetomidine, 1 or $1.5 \mathrm{mg}$ of midazolam, and $0.5 \mathrm{or} 0.75 \mathrm{mg}$ of atipamezole, assuming that body mass is 8 or $12 \mathrm{~kg}$ for adult females or males, respectively. We determined the amount of anesthetic based on data from previous captures for this population and the guidelines set by Primate Research Institute, Kyoto University (Cizauskas, 2008; Primate

130 Research Institute, Kyoto University (KUPRI), 2010). After immobilization, we sampled stomach content by inserting a Nelaton catheter from the mouth into the stomach. For colonic (rectal) microbiome, we swabbed an 8-cm sterile cotton swab into the anus. We stored all the samples in 1-ml lysis buffer (0.5\% SDS, 100 mM EDTA (pH 8.0), 100 mM Tris-HCl (pH 8.0), and $10 \mathrm{mM} \mathrm{NaCl}$ ) at room temperature. We obtained permission for the capture of macaques and entry to the study sites from the Yakushima Forest Ecosystem Conservation Center,

136 Kagoshima Prefecture, and the Ministry of Environment, Japan, adhering to the legal requirements of Japan. We followed the approved capture and sampling protocol by the Field Research Committee of Primate Research Institute, Kyoto University (KUPRI) (\#2017-009, \#2018-002 and \#2019-006). The entire project, including capture and sampling, followed the

140 Guidelines for Field Research of KUPRI and the American Society of Primatologists Principles 141 for Ethical Treatment of Non-Human Primates.

\section{Sample storage, DNA purification, 16S rRNA amplification and sequencing}

Our method followed Hayakawa et al. (2018) with slight modification. After bead-beating and centrifuging at 20,000 $\mathrm{g} g$ for $1 \mathrm{~min}$, we mixed each sample with $1000 \mu \mathrm{l}$ InhibitEX buffer

146 of the QIAamp DNA Stool Mini Kit (QIAGEN GmbH, Hilden, Germany), then centrifuged the samples at $20,000 \mathrm{x}$ g for $1 \mathrm{~min}$. After that, we mixed $600 \mu \mathrm{l}$ of the supernatant with $25 \mu \mathrm{l}$ proteinase $\mathrm{K}$ and $600 \mu \mathrm{l}$ Buffer AL. We followed the manufacturer's protocols to purify the fecal DNA. Using the Qubit dsDNA HS Assay Kit and a Qubit fluorometer (Thermo Fisher

150 Scientific), we then estimated the DNA concentration for each sample. We amplified the V3- 
151 V4 region of the 16S rRNA gene with primers as follows: S-D-Bact-0341-b-S-17 (forward) 5'CCT ACG GGN GGC WGC AG-3' and S-D-Bact-0785-a-A-21 (reverse) 5'-GAC TAC HVG GGT ATC TAA TCC-3' (Klindworth et al., 2013). To improve chastity in the Illumina

154 platform, we fused these primers with the specific overhang adapters 5'-TCG TCG GCA GCG TCA GAT GTG TAT AAG AGA CAG-[3-6-mer Ns]-[forward primer]-3' and 5'-GTC TCG TGG GCT CGG AGA TGT GTA TAA GAG ACA G-[3-6-mer Ns]-[reverse primer]-3', where the 3-6-mer Ns (NNN, NNN N, NNN NN, or NNN NNN) were in the same quantity (Lundberg, Yourstone, Mieczkowski, Jones, \& Dangl, 2013).

We purified the PCR product using Agencourt AMPure XP beads (Beckman Coulter, Inc.,

160 Carlsbad, CA, USA). Using the Illumina Nextera XT Index Kit, we attached specific dual 161 indices and sequencing adapters to each amplicon by PCR. To make the pooled sequencing library, we mixed the PCR products at the same amount of DNA (2 ng/sample). Using an Agilent 2100 Bioanalyzer (Agilent Technologies, Inc., La Jolla, CA, USA), we then estimated the fragment size distribution of the library. After diluting the library to $15 \mathrm{pM}$, we carried out the sequencing run with $30 \%$ PhiX spike-in on an Illumina MiSeq sequencing platform using the MiSeq Reagent Kit v3 (600 cycles). The read lengths from the MiSeq run were $301 \mathrm{bp}$

167 (forward sequences), $8 \mathrm{bp}$ (forward indices), $8 \mathrm{bp}$ (reverse indices), and $301 \mathrm{bp}$ (reverse sequences). We deposited the raw data in the DDBJ database with accession number DRA009571.

\section{Data analysis}

We processed the raw sequences with QIIME2-2019.10 (Bolyen et al., 2019). After demultiplexing according to the barcodes, we implemented quality control, denoising, chimera removal, and generation of amplicon sequence variants using the DADA2 pipeline (Callahan et al., 2016). The pipeline filtered out one stomach sample, UMI11, and one colonic sample, 
176 UMI21, due to low sequencing quality. We then determined phylogeny of the denoised

177 amplicon specific variants (ASVs) using the q2-fragment insertion. To assign the taxonomy of the ASVs, we used QIIME2 naïve Bayes feature classifier trained against the Greengenes 13_8 reference database. Before analysis, we excluded ASVs classified as mitochondria or

180 chloroplast from the dataset. We plotted the rarefaction curves using the "ggrare" function of 181 R package ranacapa (Kandlikar et al., 2018). To explore the functional difference between gut sites, we predicted the Kyoto Encyclopedia of Genes and Genome Orthology (KO) pathways through phylogenetic investigation of communities by reconstruction of unobserved states (PICRUSt2) (Langille et al. 2013) following guidelines at

185 https://github.com/picrust/picrust2/wiki. By default, PICRUSt2 excluded all ASVs with the nearest sequenced taxon index (NSTI) value $>2$ from the output. The average NSTI value of our dataset was $0.1901 \pm$ SD 0.1805 .

We performed statistical analyses in $\mathrm{R} v 3.6 .1$ with an alpha level of 0.05 , with $\mathrm{R}$ packages phyloseq (McMurdie \& Holmes, 2013), vegan (Oksanen et al., 2019), and microbiome (Lahti \& Shetty, 2012). For analysis, we transformed the dataset to compositional abundance (i.e. \% of total sequences per sample) using the "transform" function in package microbiome. We calculated alpha diversity through the "alpha" function in package microbiome. To test the effect of gut sites in alpha diversity (observed richness and Shannon index), we used the pairwise Wilcoxon rank sum test with P-adjustment using the false

195 discovery rate (FDR) method. For beta diversity (weighted and unweighted UniFrac), we 196 constructed principal coordinate analysis (PCoA) plots based on unweighted and weighted 197 UniFrac distances calculated using ASVs. We then used the PERMANOVA test with the 198 "adonis" function in package vegan (permutation =999). To detect the bacterial taxa and KO 199 pathways that were significantly different between stomach and colonic microbiota (log linear 200 discriminant analysis (LDA) score $>2.0, \mathrm{P}<0.05$ ), we carried out linear discriminant analysis 
201 of effect size (LEfSe) with the default parameters (Segata et al., 2011) available at

202 http://huttenhower.sph.harvard.edu/galaxy/. We conducted the LEfSe on bacterial taxa at level

2036 (i.e. genus level).

204

205 Results

Sequencing result and basic characteristics of stomach and colonic microbiome

After quality filtering, we acquired 1,296,806 reads from 9 stomach and 12 colonic

samples of Japanese macaques (Supplementary Table 1). For the stomach samples, the average

reads obtained per sample was $15,448 \pm \mathrm{SD} 13,119$. For the colon samples, the average reads

210 obtained per sample was $100,081 \pm$ SD 118,889. The rarefaction plot for the samples showed

211 that the sequencing depth was sufficient (Supplementary Figure 1).

The stomach and colon did not share any ASVs. In the colon, the 1290 ASVs uncovered

were from 14 phyla, 23 classes, 29 orders, and 46 families. The top three abundant phyla of the colonic microbiome were Firmicutes $(74.78 \pm$ SD $9.90 \%)$, Bacteroidetes (12.04 \pm SD $10.12 \%)$, and Proteobacteria (4.61 \pm SD $3.24 \%$ ) (Figure 1; Supplementary Table 2). In contrast, the top

216 three abundant phyla of the stomach microbiota were Proteobacteria, Firmicutes, and

217 Bacteroidetes, making up $70.07 \pm$ SD $17.09 \%, 20.79 \pm$ SD $13.16 \%$, and $1.96 \pm$ SD $2.29 \%$ of the stomach microbiome (Figure 1; Supplementary Table 2). The 240 ASVs uncovered in the stomach were from 6 known phyla, 12 classes, 17 orders, and 21 families.

\section{Alpha diversity and beta diversity differed significantly between stomach and colon}

Alpha diversity indices (observed richness and Shannon index) were significantly by FDR: observed richness: $V=27, P=0.0313$, Figure $2 \mathrm{a}$; Shannon index: $\mathrm{V}=28, \mathrm{P}=0.0156$, 
$30 \pm$ SD 9.23 and $2.92 \pm$ SD 0.29 , respectively. On the contrary, average observed richness and

227

228

229

230

231

232

233

234

235

236

237

238

239

240

241

242

243

Shannon index of the colonic microbiome were $119.55 \pm$ SD 109.88 and $4.16 \pm$ SD 0.86 .

PCoA plots based on unweighted (Figure 3a) and weighted UniFrac distance (Figure 3b) revealed that the samples form two distinctive clusters based on the gut site. In both plots, the colon samples were more scattered compared with the stomach samples. Adonis tests also suggested a significant effect of gut sites to the gut microbiome (Adonis: unweighted UniFrac: $\mathrm{R}^{2}=0.1029, \mathrm{P}=0.001$; weighted UniFrac: $\left.\mathrm{R}^{2}=0.2408, \mathrm{P}=0.001\right)$. Microbiota of the stomach and colon were different in both composition and abundance. The difference in microbial composition between gut sites overrode the difference caused by seasonal variation and/or identity of the individuals (i.e. troop and sex). We did not find any effect of the troop (Adonis: unweighted UniFrac: $\mathrm{R}^{2}=0.1144, \mathrm{P}=0.310$; weighted UniFrac: $\mathrm{R}^{2}=0.1306, \mathrm{P}=0.249$ ) or sex of the individuals (Adonis: unweighted UniFrac: $\mathrm{R}^{2}=0.0567, \mathrm{P}=0.354$; weighted UniFrac: $\left.\mathrm{R}^{2}=0.0308, \mathrm{P}=0.831\right)$. Since we collected the samples at different seasons/times of different years, unweighted UniFrac, but not on weighted UniFrac, was marginally significantly related to the year of collection (Adonis: unweighted UniFrac: $\mathrm{R}^{2}=0.0671, \mathrm{P}=0.048$; weighted UniFrac: $\mathrm{R}^{2}=0.0620, \mathrm{P}=0.313$ ). Close examination of datasets containing only stomach or colon samples, however, suggested little difference based on the year of collection (Adonis: stomach: $\mathrm{R}^{2}=0.1533, \mathrm{P}=0.298$; colon: $\left.\mathrm{R}^{2}=0.1023, \mathrm{P}=0.318\right)$. This marginal effect may be a result of the small sample size.

\section{Taxonomy-based comparison between stomach and colon of Japanese macaques}

Through the LEfSe test, we detected the bacterial genera whose relative abundance differs significantly between the colonic and stomach microbiomes of Japanese macaques. In total, 133 genera were significantly enriched at specific gut sites (LEfSe: $\log$ LDA score > 2.0, $\mathrm{P}<0.05$; Figure 4; Supplementary Table 3). Of these taxa, 26 were enriched in the stomach, 
251 including orders Pasteurellales and Enterobacteriales (class Gammaproteobacteria),

252 Lactobacillales and Gemellales (class Bacilli), Neisseriales (class Betaproteobacteria), and

253 Fusobacteriales (class Fusobacteriia). In the colon, 107 genera were enriched, mainly from

254 phyla Verrucomicrobia, Tenericutes, and Bacteroidetes and orders Clostridiales (phylum

255 Firmicutes) and Bacteroidales (phylum Bacteroidetes) (Supplementary Figure 2). In particular,

256 the top 15 enriched genera were mostly from families Ruminococcaceae and Lachnospiraceae

257 of the order Clostridiales.

\section{Predicted functional difference between stomach and colon}

Overall, PICRUSt2 identified 154 KO pathways (average NSTI: $0.1901 \pm$ SD 0.1805)

(Douglas et al., 2019). Based on LEfSe analysis, we defined 75 differentially abundant pathways between the stomach and colon (LEfSe: log LDA score $>2.0, \mathrm{P}<0.05$ ). Among these, 36 pathways were enriched in the colon and 39 were enriched in the stomach (Figure 5; metabolism. Specifically, the top enriched metabolic pathways in the colon microbiome were related to the metabolism of multiple nutrients such as terpenoids, polyketides, amino acid and glycan. Other than the metabolic pathways, multiple pathways related to cellular processes and genetic information processing were also enriched in the colonic microbiome. On the other hand, the stomach microbiome was especially enriched with metabolic pathways related to carbohydrates e.g. ascorbate and aldarate metabolism and citrate cycle. Furthermore, pathways related to the metabolism of other amino acids, e.g. glutathione metabolism, were enriched in the stomach microbiome. Other than metabolic pathways, stomach microbiome was also enriched in pathways related to environmental information processing, such as the phosphotransferase system and $\mathrm{ABC}$ transporters. 
Discussion

277

278

279

280

281

282

283

\section{Stomach microbiome is less diverse than colonic microbiome}

Our study found that wild Japanese macaques' stomach microbiome was less diverse than their colonic microbiome, supporting findings in the previous studies on mammals (redshanked doucs (Clayton et al., 2019), Abert's and fox squirrels (Reed, Pigage, Pigage, Glickman, \& Bono, 2019), and pikas (H. Li et al., 2017)). Such a difference in diversity revealed the strong effect exerted on the microbiota by the physiochemical environment in the stomach. The stomach generally has a rapid flow of low-pH gastric acid, causing strong disturbance for the survival and growth of microbes (Lambert, 1998; Savage, 1977). As a result, the stomach not only has lower microbial diversity but also may have lower microbial biomass than the colon. The indigenous microbes in such an environment are likely have a tolerance to the acidic and aerobic environment in the stomach and could reproduce rapidly as a way to maintain an active population in the stomach. Though not able to colonize the stomach, some microbes presumably could pass through the stomach and eventually colonize the lower GI tract, such as the colon. The colon provides a rather different environment for bacterial growth: it is characterized by an anaerobic and neutral-to-alkaline condition. Together with the extended transit time, microbes are able to establish populations and form complex interactions within the colon (Müller et al., 2019; Roager et al., 2016). In the case of humans, the halfemptying time of the colon (ca $400 \mathrm{~min}$ ) could double that of the stomach (ca $165 \mathrm{~min}$ ) (Camilleri et al., 1989). The physiochemical environment and fast transit of the stomach may present as a bottleneck for bacterial growth, "selecting" the gut microbes passing down to the lower GI tract. However, the gut microbes may then be able to establish a population once they pass through.

\section{Taxonomic difference between stomach and colon microbiome of Japanese macaques}


As adaptive characteristics to the acidic and aerobic conditions, the stomach

302 microbiome is enriched by acid- and aero-tolerant microbes. Our results revealed that

303 Proteobacteria were especially abundant in the stomach $(70.07 \%)$ in comparison with their

304 proportion in the colon, which is just 4.61\%. Unlike the majority of gut microbes,

305 Proteobacteria are often facultatively anaerobic, and thus are competitive in surviving in the

306 oxic environment of the stomach (Moon, Young, Maclean, Cookson, \& Bermingham, 2018;

307 Shin, Whon, \& Bae, 2015). By LEfSe analysis, we also identified Lactobacillales enriched in

308 the stomach microbiome. In addition to their ability to withstand an oxic condition, they are

309 also acid-tolerant, which may allow the species to flourish in the stomach (Walter, 2008).

310 Residing in the epithelial surface of the stomach, Lactobacillales species are able to maintain

311 a community even under the continuous disturbance of gastric acid (Savage, 1977; Walter,

312 2008). As opposed to the stomach microbiome, we found colonic microbiota enriched in

313 anaerobic microbes that actively involved in fiber degradation. For example, families

314 Lachnospiracea and Ruminococcaceae and genus Prevotella were more abundant in the colon.

315 These bacterial taxa are active plant degraders with key carbohydrate-active enzymes, sugar

316 transport mechanisms, and metabolic pathways (Biddle, Stewart, Blanchard, \& Leschine, 2013;

317 Chen et al., 2017). The presence of fiber-degrading bacterial taxa such as families

318 Lachnospiracea and Ruminococcaceae and genus Prevotella corroborates the major role of

319 colonic microbiota as fiber fermenters. Nevertheless, the absolute abundance of these bacterial

320 taxa would possibly be higher in the colonic microbiome if the biomass of the stomach

321 microbiome were really low.

322 Interestingly, we found that the bacterial taxa enriched in the stomach were related to

323 the oral cavity in other mammals, including humans. For example, genera Veillonella and

324 Streptococcus, the oral nitrate-reducing bacteria, are common in the mouth or throat of feral

325 horses and humans (Abranches et al., 2019; Doel, Benjamin, Hector, Rogers, \& Allaker, 2005; 
Meyer et al., 2010). Hence, the community we observed in the stomach may have represented

327 the transient microbes that were swallowed during food intake of Japanese macaques. Japanese macaques usually store food in their cheek pouch for an extended duration (Yumoto, Noma, \& Maruhashi, 1998). The microbes in the oral cavity may colonize the food surface before the macaques actually swallow the food. This partly supports the notion that the gut microbes enter from the oral cavity but then the GI sites "selects" out a part through the varied physiochemical environments. It would be interesting to further study how the microbes transfer from the oral cavity to the stomach and the lower GI tract.

\section{Functional difference between stomach and colon microbiome}

According to the functional prediction by PICRUSt2, the main functional differences between the stomach and colonic microbiomes were related to metabolism. Such differences may be related to the different digestive roles of the stomach and the colon. The stomach microbiome was more enriched in the metabolic pathways involving carbohydrates, especially

340 simple sugar. For example, we found glycolysis/gluconeogenesis and citrate cycle (TCA) enriched in the stomach microbiome. The microbes may utilize part of the simple sugar that was not digested by the enzyme in the stomach. However as mentioned above, the stomach microbiome may be less abundant and less diverse. While the stomach microbiome may have

344 functions supplementing the digestive role of the stomach, the overall effect remains limited.

345 On the other hand, metabolic pathways related to terpenoids, polyketides, amino acid and 346 glycan increased in the colonic microbiome. Glycan biosynthesis and metabolism are also 347 abundant in the gut microbiome of Tibetan macaques during winter (Sun et al., 2016). These 348 pathways are related to the digestion of glycan produced by the breakdown of cellulose and 349 hemicellulose. Since the colonic microbiome is the main fermentation site, it makes sense that 350 the enriched pathways are related to the digestive efficiency of the fibrous foods eaten by the 
351

352

353

354

355

356

357

358

macaques. Overall, the differentially enriched pathways implied that the microbial communities in both gut sites are equipped to supplement the digestive functions of these gut sites.

\section{Stomach microbiome of Japanese macaques compared to foregut microbiome of colobus} Compared with the foregut-fermenting NHPs, the relative difference in diversity between the gut sites was great in this study (Table 1). Given the biases caused by varied sampling and analysis methods across studies (Asangba et al., 2019; Hayakawa, Sawada, et al., 2018), we only made comparisons of diversity across different host species in the form of stomach to colon ratio, instead of the absolute number of ASVs or any other index. In our study, the observed richness of the macaques' stomach microbiome is nearly a quarter that of the colonic microbiome. On the other hand, the red-shanked douc's foregut microbiome is about half as diverse as the hindgut microbiome (Clayton et al., 2019). Again, this may be due to the difference in gut physiology between foregut- and hindgut-fermenting animals. The colobines are anatomically unique in having evolved a large, sacculated foregut for extended fermentation (Matsuda, Chapman, \& Clauss, 2019). Compared to the hindgut fermenters like Japanese macaques in the present study, the foregut of the colobines is relatively alkaline for the optimal fermentation condition (Lambert, 1998). The relatively alkaline stomach environment of colobines may allow a more diverse foregut microbiota and thus maximize energy harvest from their nutritionally poor folivory-based diet. Despite the biases caused by variations in sampling, storage and analysis methods across studies, the relative difference in alpha diversity indices between the foregut- and hindgut-fermenting NHPs is apparent. However, again, the current study remains preliminary, and further studies, including more species and a larger sample size, would greatly improve our knowledge of the stomach/foregut microbiome of NHPs overall. 
In comparing composition at the phylum level, the top two dominant phyla in the stomach microbiome of wild Japanese macaques and the foregut of captive red-shanked doucs (Clayton et al., 2019) were Proteobacteria and Firmicutes, different from those of the wild proboscis monkeys (Hayakawa, Nathan, et al., 2018) studied, which are dominated by Firmicutes and Bacteroidetes. Notably, the dominance of Firmicutes and Bacteroidetes rather than Proteobacteria is a more common pattern found in the colonic microbiome of mammals including NHPs (Amato et al., 2015; Clayton et al., 2018; Lee, Hayakawa, Kiyono, Yamabata, \& Hanya, 2019; Ley, Lozupone, Hamady, Knight, \& Gordon, 2008). As mentioned above, Proteobacteria are competitive in surviving the relatively oxygen-abundant environment of the stomach. The foregut of wild proboscis monkeys may present an environment similar to the colon, thus harboring a colonic microbiome-like community. Alternatively, the enriched Proteobacteria found in the stomach/foregut microbiome of Japanese macaques and redshanked doucs may be replaced by functionally redundant microbial species from the phyla Firmicutes and Bacteroidetes in the foregut microbiome of the proboscis monkeys. The difference between the foregut microbiomes of the two colobines may be related to the simplified captive diet that includes more easily digestible foods. The foregut microbiome of captive proboscis monkeys was less diverse than and compositionally different from that of the wild proboscis monkeys which forage on diverse types of plants (Hayakawa, Nathan, et al., 2018). Hence, the foregut of the captive red-shanked doucs may be different from that of proboscis monkeys through divergence in macronutrient intake. To clarify the general pattern

396 of the dominant phyla and species in the stomach/foregut microbiome as well as the related 397 factors, data from more species and a larger sample size are needed. In the present study, the 398 stomach microbiome composition of Japanese macaques was marginally related to the dietary 399 variation across seasons. However, our examination of the effect of seasons, sex and other host 400 factors remains preliminary due to the limited sample size. It would be interesting to carry out 
401

402

403

404

405

406

407

408

409

410

411

412 Accession number. The raw data is available in the DDBJ database with accession number

413

414

415

416

417

418

419

420

421

422

423

424

425

a detailed study to examine the response of the foregut/stomach microbiome to environmental factors.

\section{Conclusion}

Overall, the stomach and colonic microbiome of the Japanese macaques are distinctive from each other in diversity, composition and function. Compared with the foregut-fermenting NHPs, the stomach of hindgut-fermenting NHPs potentially present a harsher physiochemical environment for microbial acquisition and survival. Our result revealed the filtering effect imposed by different GI sites on the gut microbiome, shedding light on how microbes adapt to different physiochemical GI environments and distribute along the GI tract.

\section{DRA009571.}

Competing interests: The authors declare no competing interests.

\section{Acknowledgements}

Drs. Naofumi Nakagawa, Tatsuro Kawazoe, Yoshiyuki Tabuse, and Tianmeng He, who helped us with the capture of the animals, which followed the Guidelines for Field Research of Nonhuman Primates of the Primate Research Institute, Kyoto University (KUPRI). We obtained permission for the capture of macaques and entry to the study sites from Yakushima Forest Ecosystem Conservation Center, Kagoshima Prefecture and the Ministry of Environment, Japan, adhering to the legal requirements of Japan. We followed the approved capture and sampling protocol by Field Research Committee of KUPRI (\#2017-009, \#2018-002 and \#2019-006).

Funding information. We received financial support from the MEXT Grant-in-Aid for the Scientific Research B (17H01911) to Mieko Kiyono, TH and GH, JSPS Grant-in-Aid for 
426 Young Scientists 19K16241 to TH, Promotion of Joint International Research 15KK0256 and 427 19KK0186 to GH, as well as Grant-in-Aid for JSPS Research Fellow (16J01208) and Grant428 in-Aid for Young Scientists (18K14490) to YK.

429

$430 \quad$ References

431 Abranches, J., Zeng, L., Kajfasz, J. K., Palmer, S., Chakraborty, B., Wen, Z., ... Lemos, J. A. (2019). Biology of oral Streptococci. In Gram-Positive Pathogens (pp. 426-434). John Wiley \& Sons, Ltd. DOI: 10.1128/9781683670131.ch26

Amato, K. R., Leigh, S. R., Kent, A., Mackie, R. I., Yeoman, C. J., Stumpf, R. M., .. Garber, P. A. (2015). The gut microbiota appears to compensate for seasonal diet variation in the wild black howler monkey (Alouatta pigra). Microbial Ecology, 69(2), 434-443. DOI: $10.1007 / \mathrm{s} 00248-014-0554-7$

Amato, K. R., Metcalf, J. L., Song, S. J., Hale, V. L., Clayton, J., Ackermann, G., ... Braun, J. (2016). Using the gut microbiota as a novel tool for examining colobine primate GI health. Global Ecology and Conservation, 7, 225-237. DOI: 10.1016/j.gecco.2016.06.004

Biddle, A., Stewart, L., Blanchard, J., \& Leschine, S. (2013). Untangling the genetic basis of fibrolytic specialization by Lachnospiraceae and Ruminococcaceae in diverse gut communities. Diversity, 5(3), 627-640. DOI: 10.3390/d5030627

Bolyen, E., Rideout, J. R., Dillon, M. R., Bokulich, N. A., Abnet, C. C., Al-Ghalith, G. A., ... Caporaso, J. G. (2019). Reproducible, interactive, scalable and extensible microbiome data science using QIIME 2. Nature Biotechnology, 37(8), 852-857. DOI: 
449 Callahan, B. J., McMurdie, P. J., Rosen, M. J., Han, A. W., Johnson, A. J. A., \& Holmes, S.

450

451

452

453

454

455

456

457

458

459

460

461

462

463

464

465

466

467

468

469

470

471

472

473 P. (2016). DADA2: High-resolution sample inference from Illumina amplicon data. Nature Methods, 13(7), 581-583. DOI: 10.1038/nmeth.3869

Camilleri, M., Colemont, L. J., Phillips, S. F., Brown, M. L., Thomforde, G. M., Chapman, N., \& Zinsmeister, A. R. (1989). Human gastric emptying and colonic filling of solids characterized by a new method. The American Journal of Physiology, 257(2), G284290. DOI: 10.1152/ajpgi.1989.257.2.g284

Chen, T., Long, W., Zhang, C., Liu, S., Zhao, L., \& Hamaker, B. R. (2017). Fiber-utilizing capacity varies in Prevotella-versus Bacteroides-dominated gut microbiota. Scientific Reports, 7(1), 2594. DOI: 10.1038/s41598-017-02995-4

Cizauskas, C. A. (2008). Zoo animal \& wildlife immobilization and anesthesia. Journal of Wildlife Diseases, 44(2), 528-530. DOI: 10.7589/0090-3558-44.2.528

Clayton, J. B., Gomez, A., Amato, K., Knights, D., Travis, D. A., Blekhman, R., ... Johnson, T. J. (2018). The gut microbiome of nonhuman primates: Lessons in ecology and evolution. American Journal of Primatology, 80(6), e22867. DOI: 10.1002/ajp.22867

Clayton, J. B., Shields-Cutler, R. R., Hoops, S. L., Al-Ghalith, G. A., Sha, J. C. M., Johnson, T. J., \& Knights, D. (2019). Bacterial community structure and function distinguish gut sites in captive red-shanked doucs (Pygathrix nemaeus). American Journal of Primatology, 81(10-11), e22977. DOI: 10.1002/ajp.22977

Doel, J. J., Benjamin, N., Hector, M. P., Rogers, M., \& Allaker, R. P. (2005). Evaluation of bacterial nitrate reduction in the human oral cavity. European Journal of Oral Sciences, 113(1), 14-19. DOI: 10.1111/j.1600-0722.2004.00184.x

Douglas, G. M., Maffei, V. J., Zaneveld, J., Yurgel, S. N., Brown, J. R., Taylor, C. M., ... Langille, M. G. I. (2019). PICRUSt2: An improved and extensible approach for metagenome inference. BioRxiv. DOI: 10.1101/672295 
474 Gu, S., Chen, D., Zhang, J.-N., Lv, X., Wang, K., Duan, L.-P., ... Wu, X.-L. (2013).

475

476

477

478

479

480

481

482

483

484

485

486

487

488

489

490

491

492

493

494

495

496

497
Bacterial community mapping of the mouse gastrointestinal tract. PLOS ONE, 8(10), e74957. DOI: 10.1371/journal.pone.0074957

Hanya, G. (2004). Diet of a Japanese macaque troop in the coniferous forest of Yakushima. International Journal of Primatology, 25(1), 55-71. DOI: 10.1023/B:IJOP.0000014645.78610.32

Hanya, G., Kiyono, M., Takafumi, H., Tsujino, R., \& Agetsuma, N. (2007). Mature leaf selection of Japanese macaques: Effects of availability and chemical content. Journal of Zoology, 273(2), 140-147. DOI: 10.1111/j.1469-7998.2007.00308.x

Hanya, G., Noma, N., \& Agetsuma, N. (2003). Altitudinal and seasonal variations in the diet of Japanese macaques in Yakushima. Primates, 44(1), 51-59. DOI: 10.1007/s10329002-0007-7

Hayakawa, T., Nathan, S. K. S. S., Stark, D. J., Saldivar, D. A. R., Sipangkui, R., Goossens, B., ... Matsuda, I. (2018). First report of foregut microbial community in proboscis monkeys: Are diverse forests a reservoir for diverse microbiomes? Environmental Microbiology Reports, 10(6), 655-662. DOI: 10.1111/1758-2229.12677

Hayakawa, T., Sawada, A., Tanabe, A. S., Fukuda, S., Kishida, T., Kurihara, Y., ... Agata, K. (2018). Improving the standards for gut microbiome analysis of fecal samples: Insights from the field biology of Japanese macaques on Yakushima Island. Primates, 59(5), 423-436. DOI: 10.1007/s10329-018-0671-x

Hill, D. A. (1997). Seasonal variation in the feeding behavior and diet of Japanese macaques (Macaca fuscata yakui) in lowland forest of Yakushima. American Journal of Primatology, 43(4), 305-320. DOI: 10.1002/(SICI)1098-2345(1997)43:4<305::AIDAJP2>3.0.CO;2-0 
498 Hillman, E. T., Lu, H., Yao, T., \& Nakatsu, C. H. (2017). Microbial ecology along the

499

500

501

502

503

504

505

506

507

508

509

510

511

512

513

514

515

516

517

518

519

520 gastrointestinal tract. Microbes and Environments, 32(4), 300-313. DOI: 10.1264/jsme2.ME17017

Jandhyala, S. M., Talukdar, R., Subramanyam, C., Vuyyuru, H., Sasikala, M., \& Reddy, D. N. (2015). Role of the normal gut microbiota. World Journal of Gastroenterology, 21(29), 8787-8803. DOI: 10.3748/wjg.v21.i29.8787

Kandlikar, G. S., Gold, Z. J., Cowen, M. C., Meyer, R. S., Freise, A. C., Kraft, N. J. B., ... Curd, E. E. (2018). ranacapa: An R package and shiny web app to explore environmental DNA data with exploratory statistics and interactive visualizations. F1000Research, 7, 1734. DOI: 10.12688/f1000research.16680.1

Kurihara, Y., Kinoshita, K., Shiroishi, I., \& Hanya, G. (2020). Seasonal variation in energy balance of wild Japanese macaques (Macaca fucata yakui) in a warm-temperate forest: A preliminary assessment in the coastal forest of Yakushima. Primates, 61(3), 427-442. DOI: 10.1007/s10329-020-00797-3

Lahti, L., \& Shetty, S. (2012). Microbiome R package. Retrieved from http://microbiome.github.io/microbiome

Lambert, J. E. (1998). Primate digestion: Interactions among anatomy, physiology, and feeding ecology. Evolutionary Anthropology, 7(1), 8-20. DOI: 10.1002/(SICI)15206505(1998)7:1<8::AID-EVAN3>3.0.CO;2-C

Langille, M. G. I., Zaneveld, J., Caporaso, J. G., McDonald, D., Knights, D., Reyes, J. A., ... Huttenhower, C. (2013). Predictive functional profiling of microbial communities using 16S rRNA marker gene sequences. Nature Biotechnology, 31(9), 814-821. DOI: $10.1038 /$ nbt.2676 
521 Lee, W., Hayakawa, T., Kiyono, M., Yamabata, N., \& Hanya, G. (2019). Gut microbiota

522

523

524

525

526

527

528

529

530

531

532

533

534

535

536

537

538

539

540

541

542

543

544 composition of Japanese macaques associates with extent of human encroachment. American Journal of Primatology, 81(12), e23072. DOI: 10.1002/ajp.23072

Ley, R. E., Lozupone, C. A., Hamady, M., Knight, R., \& Gordon, J. I. (2008). Worlds within worlds: Evolution of the vertebrate gut microbiota. Nature Reviews Microbiology, 6(10), 776-788. DOI: 10.1038/nrmicro1978

Li, D., Chen, H., Zhao, J., Zhang, H., \& Chen, W. (2019). Potential functions of the gastrointestinal microbiome inhabiting the length of the rat digest tract. International Journal of Molecular Sciences, 20(5), 1232. DOI: 10.3390/ijms20051232

Li, H., Li, T., Berasategui, A., Rui, J., Zhang, X., Li, C., ... Li, X. (2017). Gut region influences the diversity and interactions of bacterial communities in pikas (Ochotona curzoniae and Ochotona daurica). FEMS Microbiology Ecology, 93(12), fix149. DOI: $10.1093 /$ femsec/fix149

Lundberg, D. S., Yourstone, S., Mieczkowski, P., Jones, C. D., \& Dangl, J. L. (2013). Practical innovations for high-throughput amplicon sequencing. Nature Methods, 10(10), 999-1002. DOI: 10.1038/nmeth.2634

Matsuda, I., Chapman, C. A., \& Clauss, M. (2019). Colobine forestomach anatomy and diet. Journal of Morphology, 280(11), 1608-1616. DOI: 10.1002/jmor.21052

McMurdie, P. J., \& Holmes, S. (2013). phyloseq: An R package for reproducible interactive analysis and graphics of microbiome census data. PLoS ONE, 8(4), e61217. DOI: 10.1371/journal.pone.0061217

Merrell, D. S., Goodrich, M. L., Otto, G., Tompkins, L. S., \& Falkow, S. (2003). PHregulated gene expression of the gastric pathogen Helicobacter pylori. Infection and Immunity, 71(6), 3529-3539. DOI: 10/frxv7z 
545

546

547

548

549

550

551

552

553

554

555

556

557

558

559

560

561

562

563

564

565

566

567

568

Meyer, W., Kacza, J., Schnapper, A., Verspohl, J., Hornickel, I., \& Seeger, J. (2010). A first report on the microbial colonisation of the equine oesophagus. Annals of Anatomy, 192(1), 42-51. DOI: 10.1016/j.aanat.2009.10.004

Moon, C. D., Young, W., Maclean, P. H., Cookson, A. L., \& Bermingham, E. N. (2018). Metagenomic insights into the roles of Proteobacteria in the gastrointestinal microbiomes of healthy dogs and cats. MicrobiologyOpen, 7(5), e00677. DOI: $10.1002 / \mathrm{mbo} 3.677$

Müller, M., Hermes, G. D. A., Canfora, E. E., Smidt, H., Masclee, A. A. M., Zoetendal, E. G., \& Blaak, E. E. (2019). Distal colonic transit is linked to gut microbiota diversity and microbial fermentation in humans with slow colonic transit. APSselect, 7(2), G361-G369. DOI: 10.1152/ajpgi.00283.2019@apsselect.2020.7.issue-2

Oksanen, J., Blanchet, F. G., Friendly, M., Kindt, R., Legendre, P., McGlinn, D., ... Wagner, H. (2019), vegan: Community ecology package. Retrieved from https://CRAN.Rproject.org/package $=$ vegan

Primate Research Institute, Kyoto University (KUPRI). (2010, June 9). Guidelines for care and use of nonhuman primates. Retrieved from https://www.pri.kyotou.ac.jp/research/sisin2010/Guidelines_for_Care_and_Use_of_Nonhuman_Primates20 100609.pdf

Reed, A., Pigage, J. C., Pigage, H. K., Glickman, C., \& Bono, J. M. (2019). Comparative analysis of microbiota along the length of the gastrointestinal tract of two tree squirrel species (Sciurus aberti and S. niger) living in sympatry. Ecology and Evolution, 9(23), 13344-13358. DOI: 10.1002/ece3.5789

Roager, H. M., Hansen, L. B. S., Bahl, M. I., Frandsen, H. L., Carvalho, V., Gøbel, R. J., ... Licht, T. R. (2016). Colonic transit time is related to bacterial metabolism and 
mucosal turnover in the gut. Nature Microbiology, 1(9), 16093. DOI: 10.1038/nmicrobiol.2016.93

571

572

573

574

575

576

577

578

579

580

581

582

583

584

585

586

587

588

589

590

591

592

593

Savage, D. C. (1977). Microbial ecology of the gastrointestinal tract. Annual Review of Microbiology, 31(1), 107-133. DOI: 10.1146/annurev.mi.31.100177.000543

Seedorf, H., Griffin, N. W., Ridaura, V. K., Reyes, A., Cheng, J., Rey, F. E., ... Woebken, D. (2014). Bacteria from diverse habitats colonize and compete in the mouse gut. Cell, 159(2), 253-266. DOI: 10.1016/j.cell.2014.09.008

Segata, N., Izard, J., Waldron, L., Gevers, D., Miropolsky, L., Garrett, W. S., \& Huttenhower, C. (2011). Metagenomic biomarker discovery and explanation. Genome Biology, 12, R60. DOI: 10.1186/gb-2011-12-6-r60

Shin, N.-R., Whon, T. W., \& Bae, J.-W. (2015). Proteobacteria: Microbial signature of dysbiosis in gut microbiota. Trends in Biotechnology, 33(9), 496-503. DOI: 10.1016/j.tibtech.2015.06.011

Stevens, C. E., \& Hume, I. D. (1998). Contributions of microbes in vertebrate gastrointestinal tract to production and conservation of nutrients. Physiological Reviews, 78(2), 393427. DOI: 10.1152/physrev.1998.78.2.393

Sun, B., Wang, X., Bernstein, S., Huffman, M. A., Xia, D.-P., Gu, Z., ... Li, J. (2016). Marked variation between winter and spring gut microbiota in free-ranging Tibetan macaques (Macaca thibetana). Scientific Reports, 6(1), 26035. DOI: $10.1038 /$ srep26035

Vega, N. M. (2019). Experimental evolution reveals microbial traits for association with the host gut. PLoS Biology, 17(2), e3000129. DOI: 10.1371/journal.pbio.3000129

Walter, J. (2008). Ecological role of Lactobacilli in the gastrointestinal tract: Implications for fundamental and biomedical research. Applied and Environmental Microbiology, 74(16), 4985-4996. DOI: 10.1128/AEM.00753-08 
594 Yumoto, T., Noma, N., \& Maruhashi, T. (1998). Cheek-pouch dispersal of seeds by Japanese 
Table 1. Foregut/stomach microbiota of the colobines and Japanese macaques

\begin{tabular}{|c|c|c|c|c|c|c|c|c|}
\hline $\begin{array}{l}\text { Common } \\
\text { name }\end{array}$ & $\begin{array}{l}\text { Scientific } \\
\text { name }\end{array}$ & Captive/Wild & $\begin{array}{l}\text { Observed } \\
\text { richness } \\
\text { (Stomach) }\end{array}$ & $\begin{array}{l}\text { Observed } \\
\text { richness } \\
\text { (Colon) }\end{array}$ & Reference & \multicolumn{3}{|c|}{ Most abundant phylum in stomach } \\
\hline $\begin{array}{l}\text { Japanse } \\
\text { macaque }\end{array}$ & $\begin{array}{l}\text { Macaca } \\
\text { fuscata }\end{array}$ & Wild & $\begin{array}{c}30.0 \pm S D \\
9.23\end{array}$ & $\begin{array}{c}119.55 \pm S D \\
109.88\end{array}$ & $\begin{array}{l}\text { Present } \\
\text { study }\end{array}$ & Proteobacteria & Firmicutes & Bacteoidetes \\
\hline $\begin{array}{l}\text { Red- } \\
\text { shanked } \\
\text { douc }\end{array}$ & $\begin{array}{l}\text { Pygathrix } \\
\text { nemaeus }\end{array}$ & Captive & $\begin{array}{c}606.5 \pm \\
166.52\end{array}$ & $\begin{array}{c}1239.5 \pm \\
146.57\end{array}$ & $\begin{array}{l}\text { Clayton et } \\
\text { al., } 2019\end{array}$ & Firmicutes & Proteobacteria & Bacteroidetes \\
\hline $\begin{array}{l}\text { Proboscis } \\
\text { monkey }\end{array}$ & $\begin{array}{l}\text { Nasalis } \\
\text { larvatus }\end{array}$ & $\begin{array}{l}\text { Captive, } \\
\text { Provisioned } \\
\text { and Wild }\end{array}$ & $501-962$ & N.A. & $\begin{array}{l}\text { Hayakawa } \\
\text { et al., } 2018\end{array}$ & Bacteroidetes & Firmicutes & Proteobacteria \\
\hline
\end{tabular}




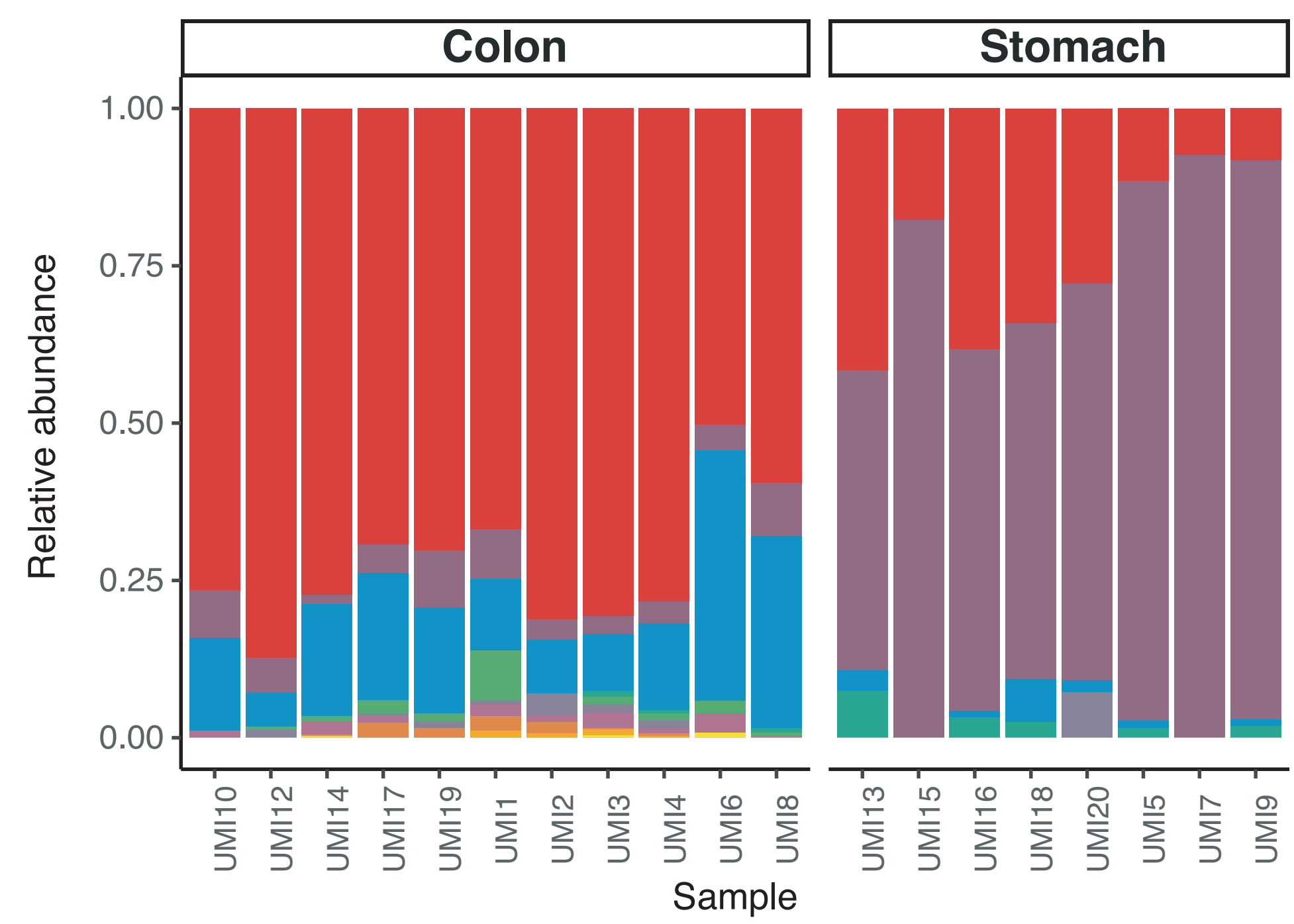

\section{Phylum}

Firmicutes

Proteobacteria

Bacteroidetes

Fusobacteria

Spirochaetes

Actinobacteria

Verrucomicrobia

Tenericutes

Lentisphaerae

Elusimicrobia

Planctomycetes

Figure 1. Relative abundance of gut bacterial taxa at phylum level (\% of total sequences per sample) 


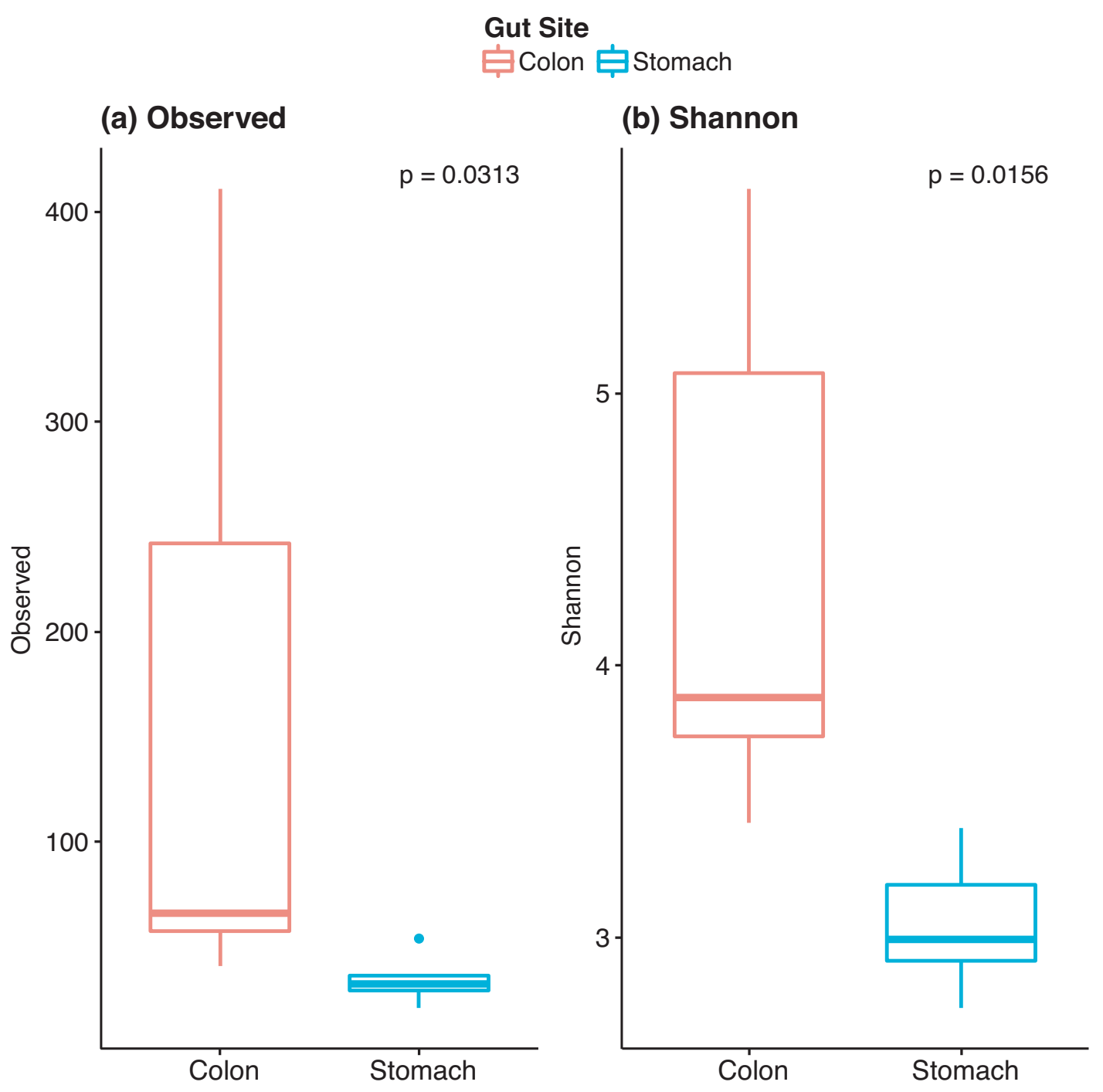

Figure 2. (a) Observed richness and (b) Shannon diversity index of stomach and colonic microbiomes of Japanese macaques 


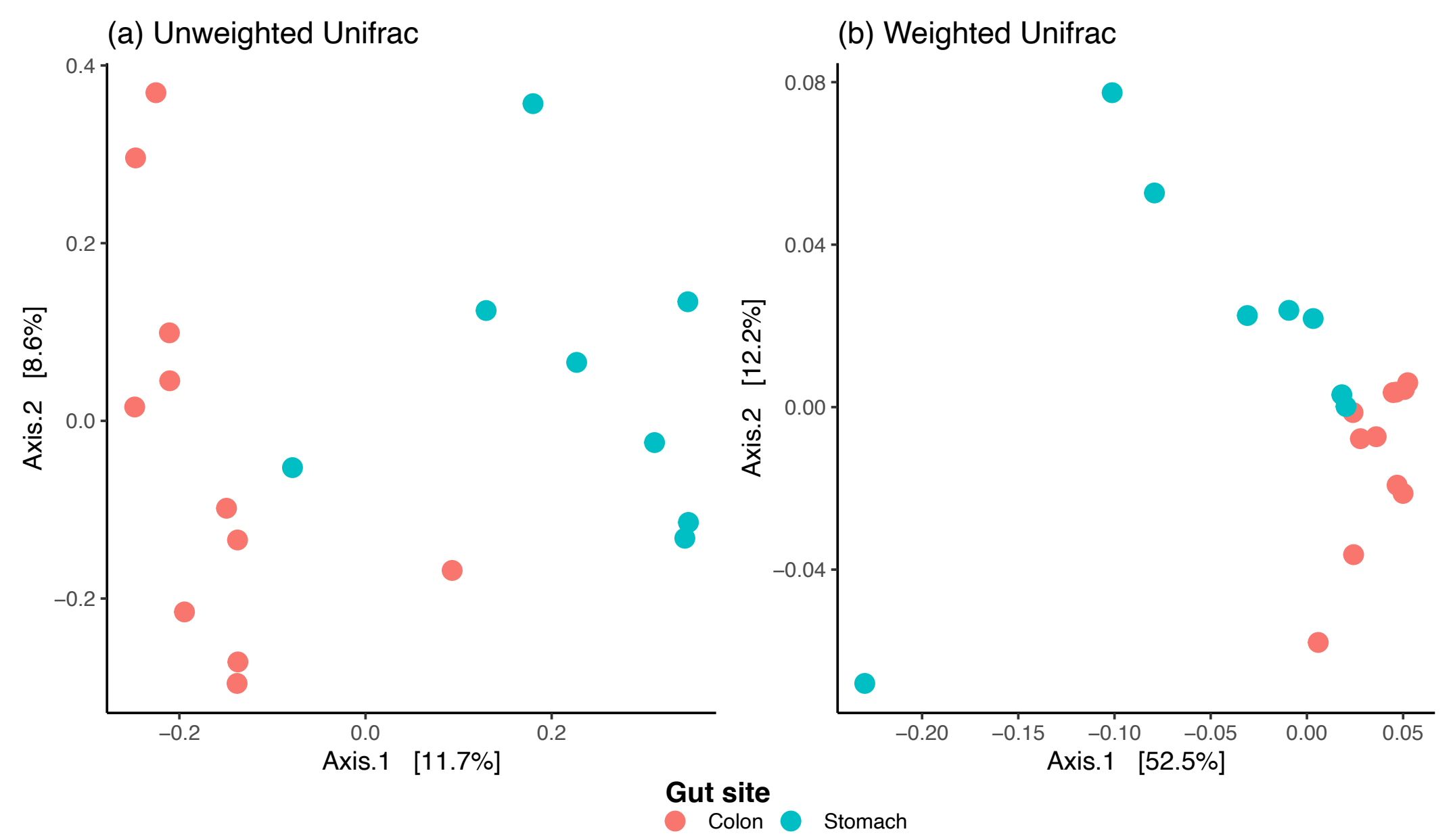

Figure 3. Principal coordinate analysis plots based on (a) unweighted and (b) weighted UniFrac distance for macaques' gut bacterial communities 


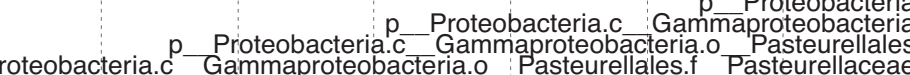

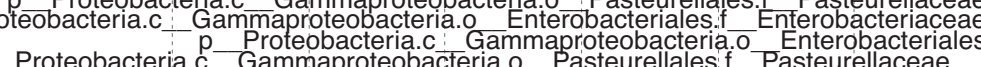

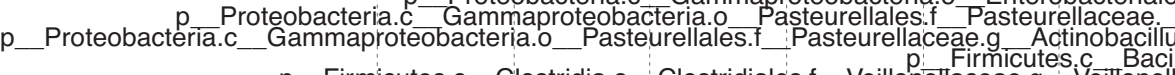

p_Firmicutes.c_Clostridia.o_Clostridiales.f_Veillonelliaceae.g Veillonel

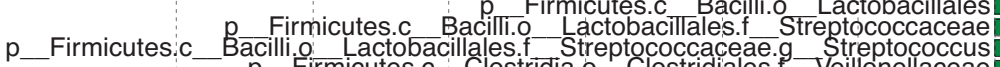

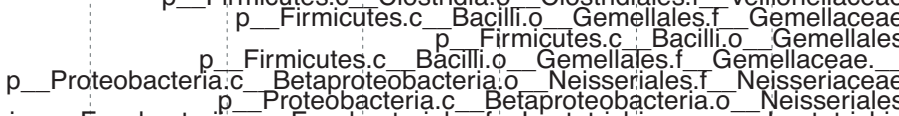
p.Fusobacteriaction

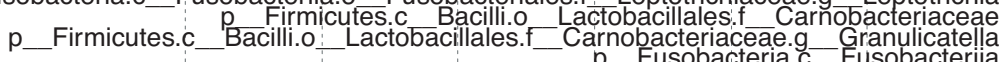

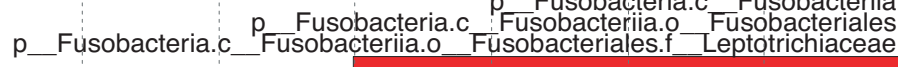

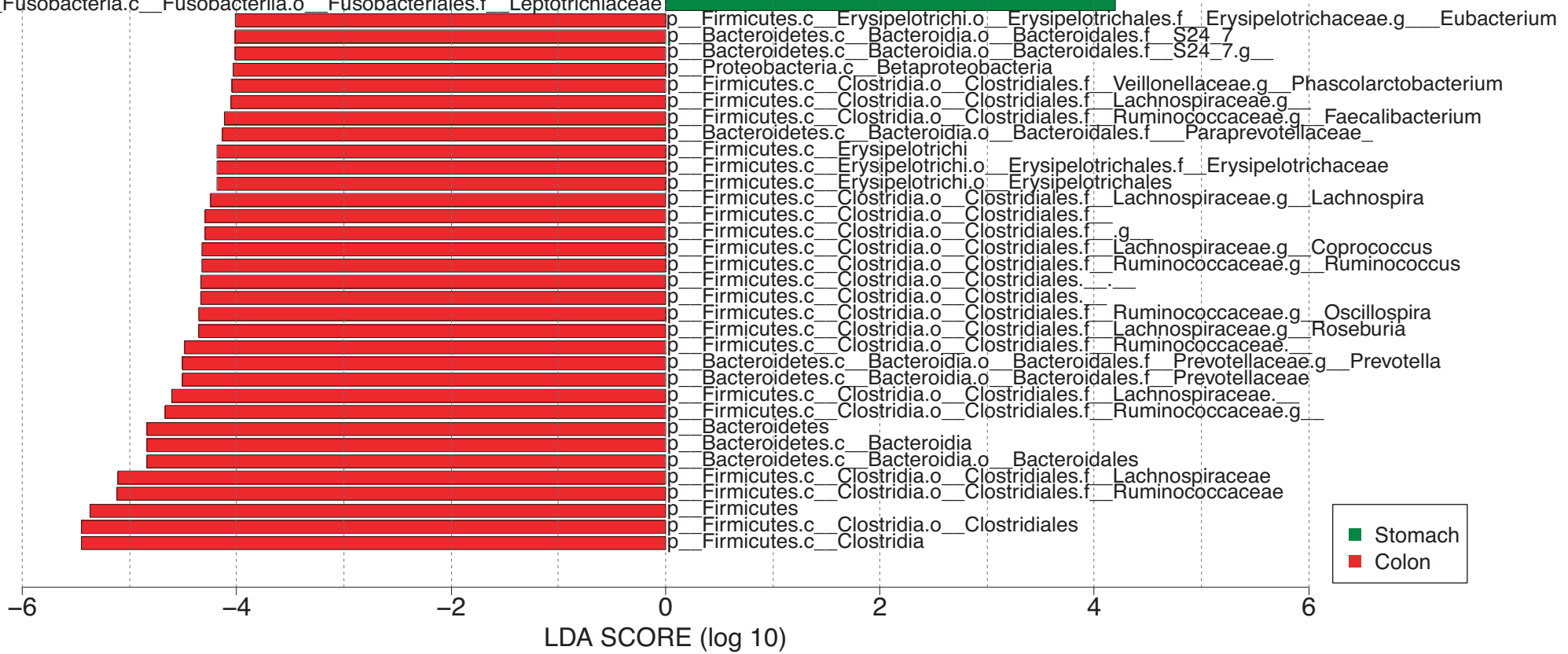

Figure 4. Gut microbial genera differentially abundant in the stomach and colonic microbiome. Plot showing the histogram of linear

discriminant analysis (LDA) scores computed for differentially abundant bacterial genera ( $\log \operatorname{LDA} \operatorname{score}>5.0, p<0.05$ ) 
ko00540. Lipopolysaccharide biosynthesis ko00130. Ubiquinone and other terpenoid-quinone biosynthesis ko00785. Lipoic acid metabolism ko00480. Glutathione metabolism ko02060. Phosphotransferase system (PTS) ko00790. Folate biosynthesis ko 0201. B i k000053. Ascorbate and aldarate metabolism ko00660.: C5-Branched dibasic acid metabolism kO04122. Sulfur relay system ko00020. Citrate cycle (TCA cycle) ko00920: Sulfur metabolism ko00450. Selenocompound metabolism ko0 0473 . D-Alanine metabolism ko00564 Glycerophospholipid metabolism ko00630. Glyoxylate and dicarboxylate metabolism k005322. Systemic lupus erythematosus ko01053. Biosynthesis of siderophore group nonribosomal peptides ko group nonribosomal peptides ko00633. Nitrotoluene degradation ko00010. Glycolysis:/ Gluconeogenesis ko00051. Fructose and mannose metabolism ko03070. Bacterial secretion system ko00520. Amino sugar and nucleotide sugar metabolism ko00440. Phosphonate and phosphinate metabolism k000350. Tyrosine metabolism

ko00562. Iniositol pho'sphate metabolism k000561. Glycerolipid metabolism ko00627: Aminobenzoate degradation ko00196. Photo'synthesis - antenna proteins ko00361. Chilorocyclohexane and chlorobenzene degradation ko05150. Staphylococcus aureus infection ko051:42. Chagas disease (American trypanosomiasis)

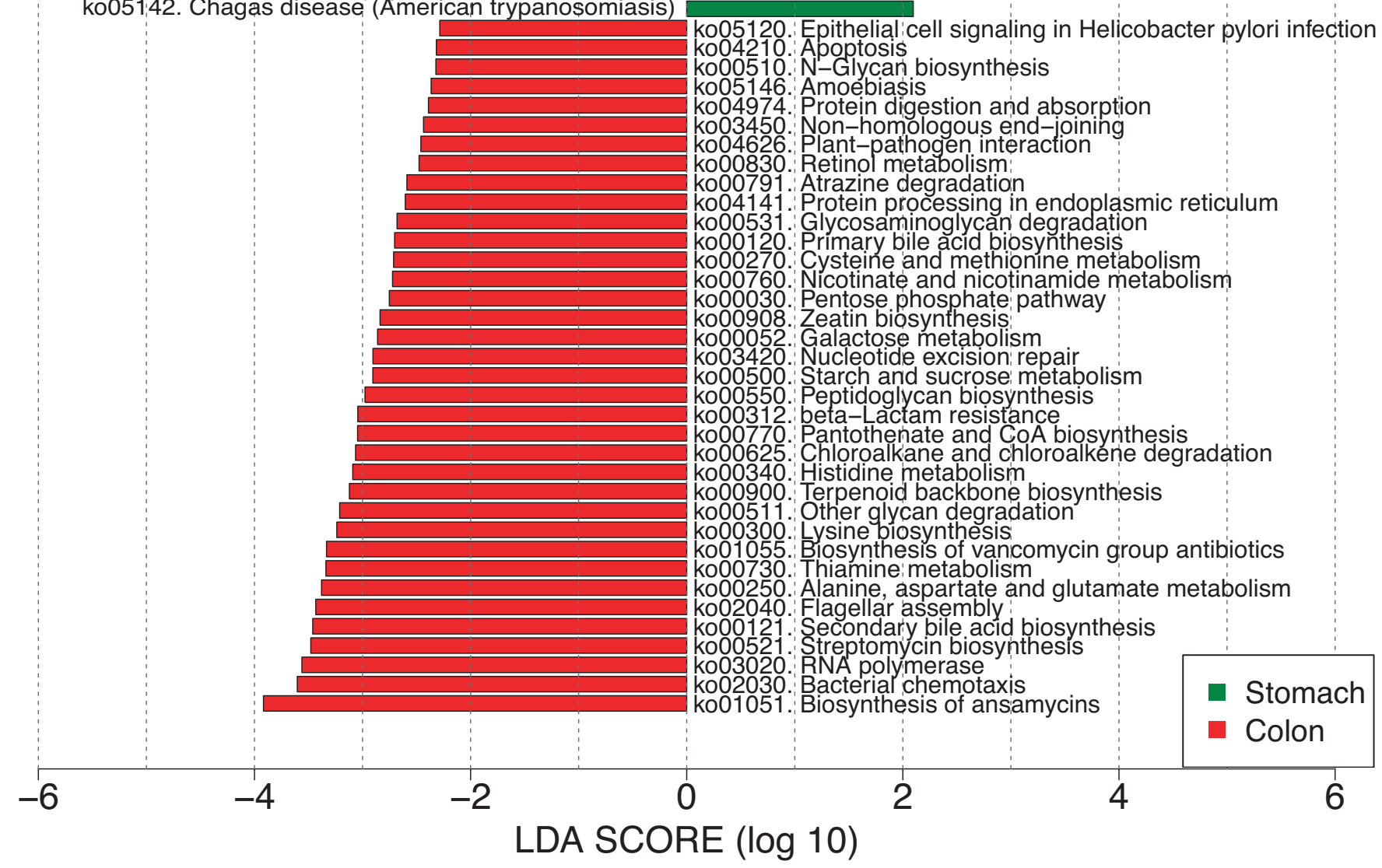

Figure 5. Histogram of LDA scores computed for differentially abundant Kyoto Encyclopedia of Genes and Genome Orthology (KO) pathways in the stomach and colonic microbiome (log LDA score $>2.0, p<0.05)$ 


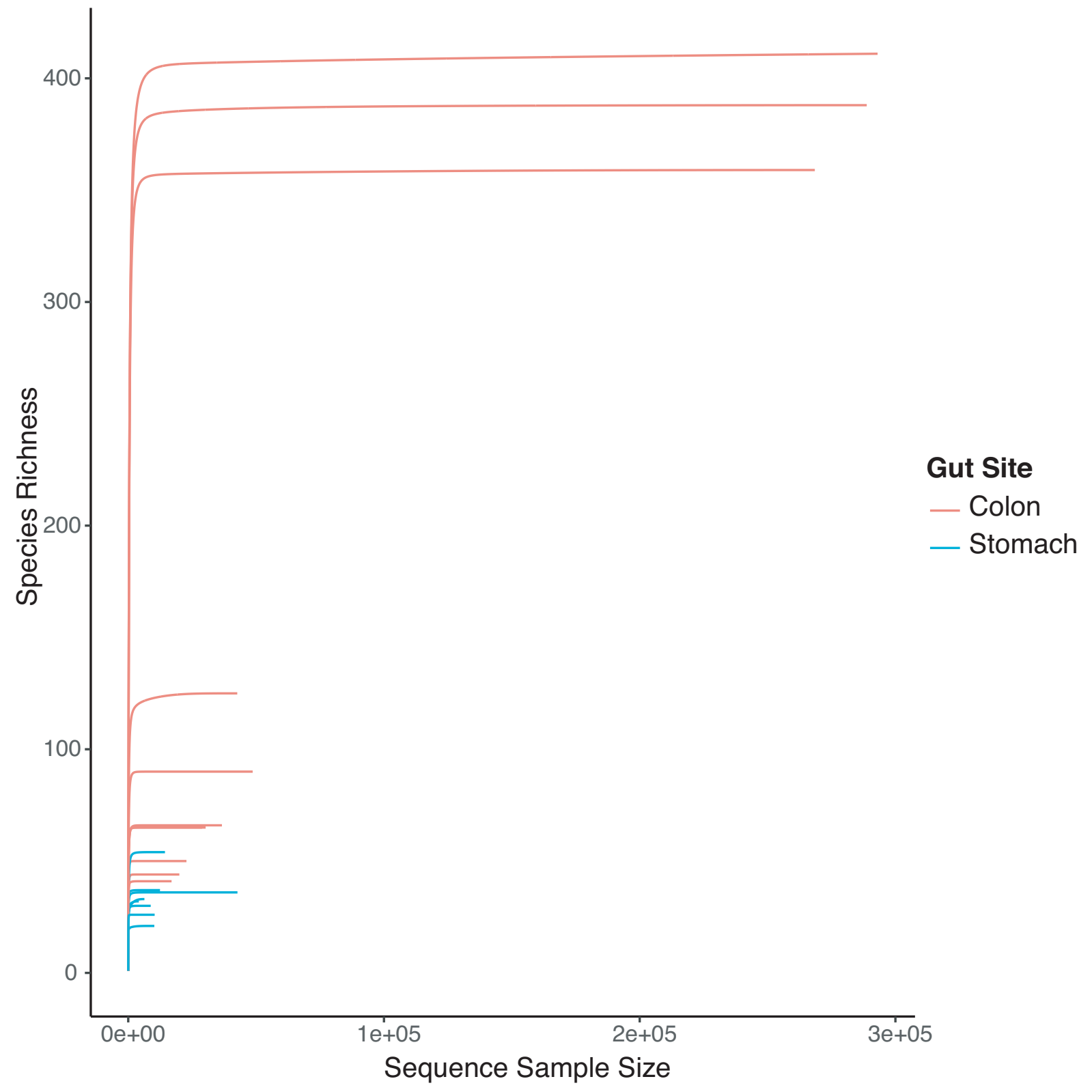

Supplementary Figure 1. Rarefaction curve of stomach and colonic samples 


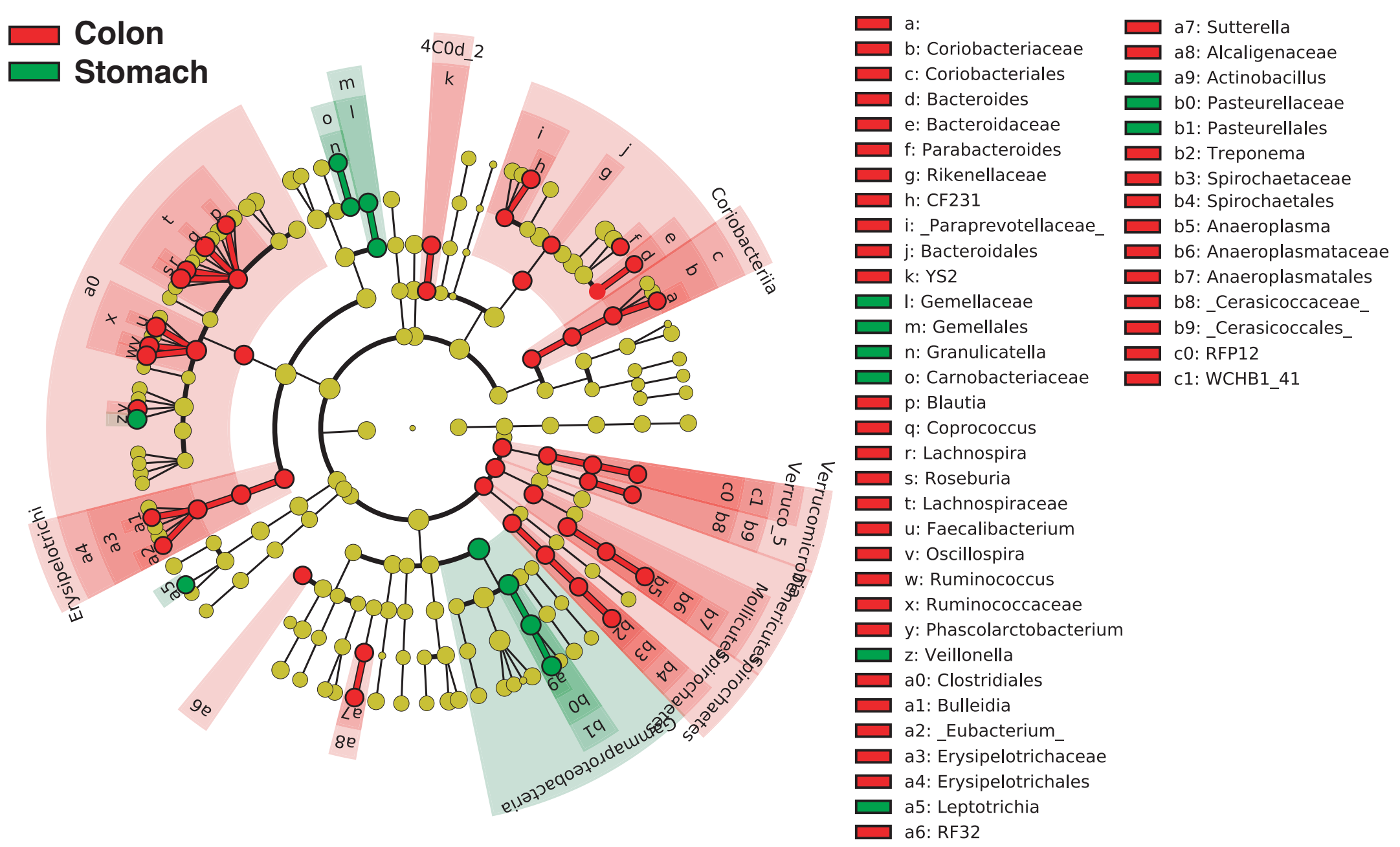

Supplementary Figure 2. Cladogram plotted from LEfSe showing the taxonomic levels represented by rings with phyla in the outermost the ring and genera in the innermost ring. Each circle is a member within that level. Those taxa in each level are colored by the gut sites in which the taxa are more abundant (log LDA score $>2.0, p<0.05)$ 
Supplementary Table 1. Sample information

\begin{tabular}{|c|c|c|c|c|c|c|}
\hline Sample ID & Group & Sex & $\begin{array}{c}\text { Collected year \& } \\
\text { month }\end{array}$ & Gut Site & PairedID & $\begin{array}{c}\text { Seuqncing } \\
\text { depth }\end{array}$ \\
\hline UMI2 & umia & female & 2017 July & colon & 2017AF & 269882 \\
\hline UMI4 & umia & female & 2018 May & colon & 2018AF & 294209 \\
\hline UMI6 & umia & male & 2018 May & colon & 2018AM & 17427 \\
\hline UMI8 & umib & female & 2018 May & colon & 2018BF & 48753 \\
\hline UMI12 & umic & female & 2018 May & colon & $2018 \mathrm{CF}$ & 23050 \\
\hline UMI14 & umic & male & 2018 May & colon & $2018 \mathrm{CM}$ & 30265 \\
\hline UMl17 & umia & male & 2019 Septmenber & colon & 2019AM & 36631 \\
\hline UMI19 & umia & female & 2019 Septmenber & colon & 2019AF & 28918 \\
\hline UMI5 & umia & female & 2018 May & stomach & 2018AF & 46398 \\
\hline UMI7 & umia & male & 2018 May & stomach & 2018AM & 4221 \\
\hline UMI16 & umia & male & 2019 Septmenber & stomach & 2019AM & 11950 \\
\hline UMI18 & umia & female & 2019 Septmenber & stomach & 2019AF & 15528 \\
\hline UMI20 & umic & male & 2019 Septmenber & stomach & $2019 \mathrm{CM}$ & 12760 \\
\hline
\end{tabular}


Supplementary Table 2. Relative abundance of microbial phyla

\begin{tabular}{|c|c|c|c|c|c|c|c|c|c|c|c|c|c|}
\hline \multirow{2}{*}{ Phylum } & \multicolumn{13}{|c|}{ Colon } \\
\hline & UMI1 & UMI10 & UMI12 & UMI14 & UMI17 & UMI19 & UMI2 & UMI3 & UMI4 & UMI6 & UMI8 & Average & SD \\
\hline Actinobacteria & $0.55 \%$ & $0.00 \%$ & $1.10 \%$ & $0.00 \%$ & $0.36 \%$ & $1.04 \%$ & $2.37 \%$ & $1.03 \%$ & $1.05 \%$ & $0.00 \%$ & $0.00 \%$ & $1.21 \%$ & $0.73 \%$ \\
\hline Bacteroidetes & $11.30 \%$ & $15.50 \%$ & $4.69 \%$ & $22.07 \%$ & $21.96 \%$ & $16.05 \%$ & $7.03 \%$ & $9.27 \%$ & $12.70 \%$ & $38.17 \%$ & $29.69 \%$ & $12.04 \%$ & $10.12 \%$ \\
\hline Cyanobacteria & $4.20 \%$ & $0.00 \%$ & $2.61 \%$ & $1.04 \%$ & $0.00 \%$ & $0.00 \%$ & $1.25 \%$ & $0.85 \%$ & $3.22 \%$ & $1.31 \%$ & $0.00 \%$ & $1.65 \%$ & $1.44 \%$ \\
\hline Elusimicrobia & $0.00 \%$ & $0.00 \%$ & $0.00 \%$ & $0.26 \%$ & $0.00 \%$ & $0.00 \%$ & $0.00 \%$ & $0.31 \%$ & $0.00 \%$ & $0.72 \%$ & $0.00 \%$ & $0.10 \%$ & $0.23 \%$ \\
\hline Euryarchaeota & $0.13 \%$ & $0.00 \%$ & $0.00 \%$ & $0.37 \%$ & $0.00 \%$ & $0.00 \%$ & $0.90 \%$ & $0.00 \%$ & $0.64 \%$ & $0.00 \%$ & $0.00 \%$ & $0.40 \%$ & $0.31 \%$ \\
\hline Firmicutes & $61.06 \%$ & $71.73 \%$ & $83.91 \%$ & $70.21 \%$ & $67.90 \%$ & $71.79 \%$ & $80.15 \%$ & $75.98 \%$ & $75.02 \%$ & $50.98 \%$ & $56.89 \%$ & $74.48 \%$ & $9.90 \%$ \\
\hline Fusobacteria & $0.00 \%$ & $0.00 \%$ & $0.00 \%$ & $0.00 \%$ & $0.00 \%$ & $0.00 \%$ & $0.00 \%$ & $0.73 \%$ & $0.41 \%$ & $0.00 \%$ & $0.67 \%$ & $0.33 \%$ & $0.29 \%$ \\
\hline Lentisphaerae & $0.89 \%$ & $0.00 \%$ & $0.00 \%$ & $0.00 \%$ & $0.00 \%$ & $0.00 \%$ & $0.52 \%$ & $0.71 \%$ & $0.20 \%$ & $0.00 \%$ & $0.00 \%$ & $0.40 \%$ & $0.33 \%$ \\
\hline OD1 & $0.00 \%$ & $0.00 \%$ & $0.00 \%$ & $0.00 \%$ & $0.00 \%$ & $0.00 \%$ & $0.00 \%$ & $0.02 \%$ & $0.00 \%$ & $0.00 \%$ & $0.00 \%$ & $0.01 \%$ & $0.01 \%$ \\
\hline Planctomycetes & $0.00 \%$ & $0.00 \%$ & $0.00 \%$ & $0.00 \%$ & $0.00 \%$ & $0.00 \%$ & $0.00 \%$ & $0.00 \%$ & $0.00 \%$ & $0.00 \%$ & $0.00 \%$ & $0.00 \%$ & $0.00 \%$ \\
\hline Proteobacteria & $7.29 \%$ & $11.80 \%$ & $7.20 \%$ & $3.38 \%$ & $4.36 \%$ & $8.67 \%$ & $3.55 \%$ & $4.18 \%$ & $3.54 \%$ & $3.96 \%$ & $11.79 \%$ & $4.61 \%$ & $3.24 \%$ \\
\hline Spirochaetes & $6.53 \%$ & $0.00 \%$ & $0.49 \%$ & $0.72 \%$ & $2.12 \%$ & $1.07 \%$ & $0.06 \%$ & $0.84 \%$ & $0.97 \%$ & $1.98 \%$ & $0.65 \%$ & $0.94 \%$ & $1.83 \%$ \\
\hline Tenericutes & $4.34 \%$ & $0.00 \%$ & $0.00 \%$ & $0.11 \%$ & $2.34 \%$ & $1.38 \%$ & $2.19 \%$ & $1.84 \%$ & $0.60 \%$ & $0.00 \%$ & $0.18 \%$ & $1.47 \%$ & $1.40 \%$ \\
\hline Verrucomicrobia & $1.47 \%$ & $0.97 \%$ & $0.00 \%$ & $1.86 \%$ & $0.96 \%$ & $0.00 \%$ & $0.87 \%$ & $2.02 \%$ & $0.62 \%$ & $2.88 \%$ & $0.14 \%$ & $1.12 \%$ & $0.92 \%$ \\
\hline WPS-2 & $0.00 \%$ & $0.00 \%$ & $0.00 \%$ & $0.00 \%$ & $0.00 \%$ & $0.00 \%$ & $0.00 \%$ & $0.00 \%$ & $0.66 \%$ & $0.00 \%$ & $0.00 \%$ & $0.18 \%$ & $0.20 \%$ \\
\hline NA & $2.24 \%$ & $0.00 \%$ & $0.00 \%$ & $0.00 \%$ & $0.00 \%$ & $0.00 \%$ & $1.13 \%$ & $2.22 \%$ & $0.36 \%$ & $0.00 \%$ & $0.00 \%$ & $1.04 \%$ & $0.90 \%$ \\
\hline
\end{tabular}




\begin{tabular}{|c|c|c|c|c|c|c|c|c|c|c|}
\hline \multirow{2}{*}{ Phylum } & \multicolumn{9}{|c|}{ Stomach } & \multirow[b]{2}{*}{ SD } \\
\hline & UMI13 & UMI15 & UMI16 & UMI18 & UMI20 & UMI5 & UMI7 & UMI9 & Average & \\
\hline Actinobacteria & $0.00 \%$ & $0.00 \%$ & $0.00 \%$ & $0.00 \%$ & $7.22 \%$ & $0.00 \%$ & $0.00 \%$ & $0.00 \%$ & $0.67 \%$ & $2.55 \%$ \\
\hline Bacteroidetes & $3.13 \%$ & $0.00 \%$ & $0.93 \%$ & $6.91 \%$ & $1.86 \%$ & $1.12 \%$ & $0.00 \%$ & $0.78 \%$ & $1.96 \%$ & $2.29 \%$ \\
\hline Cyanobacteria & $0.00 \%$ & $0.00 \%$ & $0.00 \%$ & $0.00 \%$ & $0.00 \%$ & $0.00 \%$ & $0.00 \%$ & $0.00 \%$ & $0.00 \%$ & $0.00 \%$ \\
\hline Elusimicrobia & $0.00 \%$ & $0.00 \%$ & $0.00 \%$ & $0.00 \%$ & $0.00 \%$ & $0.00 \%$ & $0.00 \%$ & $0.00 \%$ & $0.00 \%$ & $0.00 \%$ \\
\hline Euryarchaeota & $0.00 \%$ & $0.00 \%$ & $0.00 \%$ & $0.00 \%$ & $0.00 \%$ & $0.00 \%$ & $0.00 \%$ & $0.00 \%$ & $0.00 \%$ & $0.00 \%$ \\
\hline Firmicutes & $39.47 \%$ & $18.77 \%$ & $33.84 \%$ & $34.18 \%$ & $27.77 \%$ & $11.40 \%$ & $7.46 \%$ & $6.13 \%$ & $20.79 \%$ & $13.16 \%$ \\
\hline Fusobacteria & $6.99 \%$ & $0.00 \%$ & $2.86 \%$ & $2.47 \%$ & $0.00 \%$ & $1.53 \%$ & $0.00 \%$ & $1.38 \%$ & $2.16 \%$ & $2.34 \%$ \\
\hline Lentisphaerae & $0.00 \%$ & $0.00 \%$ & $0.00 \%$ & $0.00 \%$ & $0.00 \%$ & $0.00 \%$ & $0.00 \%$ & $0.00 \%$ & $0.00 \%$ & $0.00 \%$ \\
\hline OD1 & $0.00 \%$ & $0.00 \%$ & $0.00 \%$ & $0.00 \%$ & $0.00 \%$ & $0.52 \%$ & $0.00 \%$ & $0.00 \%$ & $0.21 \%$ & $0.19 \%$ \\
\hline Planctomycetes & $0.00 \%$ & $0.00 \%$ & $0.00 \%$ & $0.00 \%$ & $0.00 \%$ & $0.00 \%$ & $0.00 \%$ & $0.00 \%$ & $0.00 \%$ & $0.00 \%$ \\
\hline Proteobacteria & $45.12 \%$ & $81.23 \%$ & $50.85 \%$ & $56.44 \%$ & $63.10 \%$ & $85.10 \%$ & $92.54 \%$ & $66.41 \%$ & $70.07 \%$ & $17.09 \%$ \\
\hline Spirochaetes & $0.00 \%$ & $0.00 \%$ & $0.00 \%$ & $0.00 \%$ & $0.00 \%$ & $0.00 \%$ & $0.00 \%$ & $0.00 \%$ & $0.00 \%$ & $0.00 \%$ \\
\hline Tenericutes & $0.00 \%$ & $0.00 \%$ & $0.00 \%$ & $0.00 \%$ & $0.00 \%$ & $0.00 \%$ & $0.00 \%$ & $0.00 \%$ & $0.00 \%$ & $0.00 \%$ \\
\hline Verrucomicrobia & $0.00 \%$ & $0.00 \%$ & $0.00 \%$ & $0.00 \%$ & $0.00 \%$ & $0.00 \%$ & $0.00 \%$ & $0.00 \%$ & $0.00 \%$ & $0.00 \%$ \\
\hline WPS-2 & $0.00 \%$ & $0.00 \%$ & $0.00 \%$ & $0.00 \%$ & $0.00 \%$ & $0.00 \%$ & $0.00 \%$ & $0.00 \%$ & $0.00 \%$ & $0.00 \%$ \\
\hline NA & $5.29 \%$ & $0.00 \%$ & $11.51 \%$ & $0.00 \%$ & $0.05 \%$ & $0.33 \%$ & $0.00 \%$ & $25.31 \%$ & $4.14 \%$ & $9.07 \%$ \\
\hline
\end{tabular}


Supplementary Table 3. Bacterial genera identified by LEfSe analysis different between stomach and colonic microbiota (log LDA score $>2.0, p<0.05)$

\begin{tabular}{|c|c|c|c|}
\hline Differentially abundant taxa & Class & $\begin{array}{l}\log _{10}(\text { LDA } \\
\text { score) }\end{array}$ & $p$-value \\
\hline p__Firmicutes.c__Clostridia & colon & 5.4511 & 0.0003 \\
\hline p__Firmicutes.c_Clostridia.o__Clostridiales & colon & 5.4511 & 0.0003 \\
\hline p_Firmicutes & colon & 5.3684 & 0.0003 \\
\hline p__Firmicutes.c__Clostridia.o__Clostridiales.f__Ruminococcaceae & colon & 5.1202 & 0.0002 \\
\hline p_Firmicutes.c_Clostridia.o__Clostridiales.f__Lachnospiraceae & colon & 5.1121 & 0.0002 \\
\hline p__Bacteroidetes.c_Bacteroidia.o__Bacteroidales & colon & 4.8410 & 0.0004 \\
\hline p__Bacteroidetes.c__Bacteroidia & colon & 4.8410 & 0.0004 \\
\hline p__Bacteroidetes & colon & 4.8357 & 0.0004 \\
\hline p__Firmicutes.c__Clostridia.o__Clostridiales.f__Ruminococcaceae.g__ & colon & 4.6670 & 0.0002 \\
\hline p_Firmicutes.c_Clostridia.o__Clostridiales.f__Lachnospiraceae.__ & colon & 4.6074 & 0.0002 \\
\hline p__Bacteroidetes.c__Bacteroidia.o__Bacteroidales.f_Prevotellaceae & colon & 4.5093 & 0.0015 \\
\hline p__Bacteroidetes.c_Bacteroidia.o__Bacteroidales.f__Prevotellaceae.g__Prevotella & colon & 4.5073 & 0.0015 \\
\hline p__Firmicutes.c_Clostridia.o__Clostridiales.f__Ruminococcaceae.__ & colon & 4.4849 & 0.0002 \\
\hline p_Firmicutes.c__Clostridia.o__Clostridiales.f__Lachnospiraceae.g_Roseburia & colon & 4.3561 & 0.0005 \\
\hline p__Firmicutes.c__Clostridia.o__Clostridiales.f__Ruminococcaceae.g_Oscillospira & colon & 4.3527 & 0.0013 \\
\hline p_Firmicutes.c__Clostridia.o__Clostridiales.__ & colon & 4.3365 & 0.0002 \\
\hline p__Firmicutes.c__Clostridia.o__Clostridiales.__.__ & colon & 4.3365 & 0.0002 \\
\hline p_Firmicutes.c_Clostridia.o__Clostridiales.f_Ruminococcaceae.g_Ruminococcus & colon & 4.3207 & 0.0005 \\
\hline p_Firmicutes.c__Clostridia.o__Clostridiales.f__Lachnospiraceae.g__Coprococcus & colon & 4.3207 & 0.0013 \\
\hline p__Firmicutes.c_Clostridia.o__Clostridiales.f_.g_ & colon & 4.2950 & 0.0005 \\
\hline p__Firmicutes.c_Clostridia.o__Clostridiales.f_ & colon & 4.2950 & 0.0005 \\
\hline p_Firmicutes.c_Clostridia.o__Clostridiales.f__Lachnospiraceae.g_Lachnospira & colon & 4.2466 & 0.0002 \\
\hline
\end{tabular}


p__Firmicutes.c_Erysipelotrichi.o__Erysipelotrichales

p__Firmicutes.c_Erysipelotrichi.o_Erysipelotrichales.f_Erysipelotrichaceae

p__Firmicutes.c_Erysipelotrichi

p__Bacteroidetes.c_Bacteroidia.o_Bacteroidales.f__Paraprevotellaceae_

p_Firmicutes.c_Clostridia.o__Clostridiales.f_Ruminococcaceae.g_Faecalibacterium

p_Firmicutes.c_Clostridia.o__Clostridiales.f__Lachnospiraceae.g_

p__Firmicutes.c_Clostridia.o__Clostridiales.f_Veillonellaceae.g_Phascolarctobacterium

p__Proteobacteria.c__Betaproteobacteria

p__Bacteroidetes.c_Bacteroidia.o__Bacteroidales.f_S24_7.g__

p_Bacteroidetes.C_Bacteroidia.o_Bacteroidales.f_S24_7

p__Firmicutes.c_Erysipelotrichi.o__Erysipelotrichales.f_Erysipelotrichaceae.g_Eubacterium_

p_Bacteroidetes.c_Bacteroidia.o_Bacteroidales.f__Paraprevotellaceae_.g___Prevotella_

p_Cyanobacteria.c_4COd_2.o__YS2.f__

p__Cyanobacteria

p__Cyanobacteria.c_4COd_2.o__YS2.f_.g_

p__Cyanobacteria.c_4COd_2

p__Cyanobacteria.c_4COd_2.o__YS2

p__Bacteroidetes.c_Bacteroidia.o_Bacteroidales.f__Paraprevotellaceae_.g__

p__Spirochaetes

p__Firmicutes.c_Clostridia.o__Clostridiales.f_Christensenellaceae.g__

p__Firmicutes.c__Clostridia.o__Clostridiales.f_Christensenellaceae

p__Firmicutes.c_Erysipelotrichi.o__Erysipelotrichales.f_Erysipelotrichaceae.g__

p__Tenericutes

p__Bacteroidetes.c_Bacteroidia.o_Bacteroidales.__.

p_Bacteroidetes.c_Bacteroidia.o__Bacteroidales.

p__Spirochaetes.c__Spirochaetes colon

colon

colon

colon

colon

colon

colon

colon

colon

colon

colon

colon

colon

colon

colon

colon

colon

colon

colon

colon

colon

colon

colon

colon

colon

colon $\begin{array}{ll}4.1820 & 0.0002\end{array}$

$4.1820 \quad 0.0002$

$\begin{array}{ll}4.1820 & 0.0002\end{array}$

$4.1328 \quad 0.0005$

$4.1131 \quad 0.0005$

$4.0554 \quad 0.0013$

$4.0432 \quad 0.0002$

$\begin{array}{ll}4.0296 & 0.0277\end{array}$

$\begin{array}{ll}4.0184 & 0.0002\end{array}$

$4.0184 \quad 0.0002$

$\begin{array}{ll}4.0108 & 0.0331\end{array}$

$3.9777 \quad 0.0033$

$3.9256 \quad 0.0076$

$3.9256 \quad 0.0076$

$3.9256 \quad 0.0076$

$3.9256 \quad 0.0076$

$3.9256 \quad 0.0076$

$3.9231 \quad 0.0163$

$3.9203 \quad 0.0005$

$3.9092 \quad 0.0076$

$3.9092 \quad 0.0076$

$3.9026 \quad 0.0013$

$3.8969 \quad 0.0033$

$3.8807 \quad 0.0013$

$3.8807 \quad 0.0013$

$3.8774 \quad 0.0033$ 
p__Spirochaetes.c_Spirochaetes.o__Spirochaetales.f_Spirochaetaceae.g_Treponema

colon

3.8774

0.0033

p__Spirochaetes.c__Spirochaetes.o_Spirochaetales

p__Spirochaetes.c_Spirochaetes.o__Spirochaetales.f_Spirochaetaceae

colon

3.8774

0.0033

p__Proteobacteria.c__Betaproteobacteria.o_Burkholderiales

colon

$3.8774 \quad 0.0033$

p__Proteobacteria.c__Betaproteobacteria.o_Burkholderiales.

colon

3.8751

0.0013

p__Proteobacteria.c__Betaproteobacteria.o_B_Burkholderiales.

colon

$3.8604 \quad 0.0331$

p__Firmicutes.c_Clostridia.o__Clostridiales.f_Lachnospiraceae.g__Dorea

colon

$3.8603 \quad 0.0331$

p__ Verrucomicrobia

p__Bacteroidetes.c_Bacteroidia.o_Bacteroidales.f__

p_Bacteroidetes.c_Bacteroidia.o_Bacteroidales.f_.g_

p__Proteobacteria.c__Betaproteobacteria.o_Burkholderiales.f__Alcaligenaceae.g_Sutterella

colon

$3.8424 \quad 0.0076$

p__Proteobacteria.c__Betaproteobacteria.o_Burkholderiales.f_Alcaligenaceae

colon

3.8424

colon

3.8352

0.0013

$3.8300 \quad 0.0033$

$3.8300 \quad 0.0033$

$3.8294 \quad 0.0013$

p_Firmicutes.c_Clostridia.o__Clostridiales.f_Lachnospiraceae.g_Blautia

colon

colon

3.8294

p__Proteobacteria.c__Gammaproteobacteria.o__Aeromonadales.f_Succinivibrionaceae

colon

3.8272

0.0013

p__Proteobacteria.c__Gammaproteobacteria.o__Aeromonadales.f_Succinivibrionaceae.g_Succinivibrio

p__Proteobacteria.c__Gammaproteobacteria.o__Aeromonadales

p__Proteobacteria.c_Epsilonproteobacteria.o__Campylobacterales.f__Helicobacteraceae.g_Flexispira

p__Proteobacteria.c__Alphaproteobacteria.o__Rickettsiales.f__.g_

p__Proteobacteria.c__Alphaproteobacteria.o__Rickettsiales.f__

colon

3.8051

0.0033

colon

3.8051

0.0033

colon

3.8051

0.0033

colon

3.7996

0.0033

colon

3.7749

0.0163

p__Bacteroidetes.c_Bacteroidia.o_Bacteroidales.f_Bacteroidaceae.g__Bacteroides

colon

3.7749

0.0331

p__Verrucomicrobia.c__Opitutae

p__Firmicutes.c_Clostridia.o__Clostridiales.f_Clostridiaceae

colon

3.7680

0.0331

colon

0.0331

p__Bacteroidetes.c__Bacteroidia.o_Bacteroidales.f_Bacteroidaceae

colon

3.7645

0.0331

p__Actinobacteria.c__Coriobacteriia.o__Coriobacteriales.f_Coriobacteriaceae.g_

colon

3.7611

0.0076

p__Firmicutes.c_Erysipelotrichi.o__Erysipelotrichales.f_Erysipelotrichaceae.g_RFN20

colon

3.7577

0.0331

p__Verrucomicrobia.c_Opitutae.o___Cerasicoccales_

colon

3.7569

0.0163

3.7558

0.0331

colon

3.7534

0.0331 
p__Verrucomicrobia.c_Opitutae.o__Cerasicoccales_f__Cerasicoccaceae_.g_

colon

3.7534

0.0331

p__Verrucomicrobia.c_Opitutae.o

Cerasicoccales_.f

Cerasicoccaceae

colon

$3.7534 \quad 0.0331$

$\mathrm{p}$ __Actinobacteria.c__Coriobacteriia.o__Coriobacteriales.f_Coriobacteriaceae

colon

$3.7450 \quad 0.0163$

p__Actinobacteria.c__Coriobacteriia

colon

$3.7450 \quad 0.0163$

P__Actinobacteria.c__Coriobacteriia.o__Coriobacteriales

$3.7450 \quad 0.0163$

p_Proteobacteria.c_Alphaproteobacteria

colon

$3.7305 \quad 0.0163$

p__Proteobacteria.c__Alphaproteobacteria

colon

0.0163

p__Proteobacteria.c__Alphaproteobacteria.

p__Tenericutes.c__Mollicutes

p_Bacteroidetes.c_Bacteroidia.o_Bacteroidales.f__Paraprevotellaceae_.g_CF231

colon

3.7305

3.7305

0.0163

p__Bacteroidetes.c_Bacteroidia.o_Bacteroidales.f_Rikenellaceae

colon

$3.7230 \quad 0.0076$

p__Bacteroidetes.c_Bacteroidia.o__Bacteroidales.f_Rikenellaceae.g__

colon

$3.7057 \quad 0.0076$

p__Firmicutes.c_Clostridia.o__Clostridiales.f__Mogibacteriaceae_

$3.7040-0.0033$

p__Firmicutes.c__Clostridia.o__Clostridiales.f__Mogibacteriaceae_.g_

colon

$3.7040 \quad 0.0033$

p__Tenericutes.c__Mollicutes.o__Anaeroplasmatales

p__Tenericutes.c__Mollicutes.o__Anaeroplasmatales.f_Anaeroplasmataceae

p__Bacteroidetes.c_Bacteroidia.o_Bacteroidales.f_Porphyromonadaceae.g_Parabacteroides

colon

3.69910 .0076

colon

0.0076

colon

3.6991

0.0076

p__Tenericutes.c_RF3.o_ML615J_28.f_

$\mathrm{p} \_$_Tenericutes.c__RF3

p_Tenericutes.c_RF3.0_ML615J_28.f_.g_

colon

3.6984

3.6984

0.0076

colon

3.6944

0.0076

p__Tenericutes.c_RF3.0_ML615J_28

p__Verrucomicrobia.c_Verruco_5

p_Verrucomicrobia.c_Verruco_5.o_WCHB1_41.f_RFP12

p_Verrucomicrobia.c_Verruco_5.o_WWCHB1_41.f_RFP12.g__

colon

3.6869

0.0331

colon

3.6869

0.0331

colon

3.6869

0.0331

colon

3.6869

0.0331

colon

3.6757

0.0033

p_Verrucomicrobia.c_Verruco_5.o_WCHB1_41

colon

3.6757

0.0033

p__Proteobacteria.c__Alphaproteobacteria.o_RF32

colon

3.6757

0.0033

colon

3.6757

0.0033

colon

3.6503

0.0033 
p__Proteobacteria.c__Alphaproteobacteria.o_RF32.f_.g_

p__Proteobacteria.c__Alphaproteobacteria.o_RF32.f__

p__Firmicutes.c_Clostridia.o__Clostridiales.f_Veillonellaceae.g_Dialister

p__Tenericutes.c_Mollicutes.o__Anaeroplasmatales.f_Anaeroplasmataceae.g__

p__Firmicutes.c_Erysipelotrichi.o_Erysipelotrichales.f_Erysipelotrichaceae.g__Bulleidia

p_Bacteroidetes.c_Bacteroidia.o_Bacteroidales.f_RF16.g_

p__Bacteroidetes.c_Bacteroidia.o__Bacteroidales.f_RF16

p__Proteobacteria

p__Proteobacteria.c__Gammaproteobacteria

p_Proteobacteria.c__Gammaproteobacteria.o_Pasteurellales

p__Proteobacteria.c__Gammaproteobacteria.o__Pasteurellales.f__Pasteurellaceae

p__Proteobacteria.c__Gammaproteobacteria.o_Enterobacteriales.f_Enterobacteriaceae

p__Proteobacteria.c__Gammaproteobacteria.o__Enterobacteriales

p__Proteobacteria.c__Gammaproteobacteria.o__Pasteurellales.f__Pasteurellaceae.__

p__Proteobacteria.c__Gammaproteobacteria.o__Pasteurellales.f__Pasteurellaceae.g_Actinobacillus

p__Firmicutes.c_Bacilli

p__Firmicutes.c__Clostridia.o__Clostridiales.f_Veillonellaceae.g_Veillonella

p__Firmicutes.c__Bacilli.o__Lactobacillales

p__Firmicutes.c__Bacilli.o__Lactobacillales.f__Streptococcaceae

p__Firmicutes.c_Bacilli.o__Lactobacillales.f__Streptococcaceae.g_Streptococcus

p__Firmicutes.c__Clostridia.o__Clostridiales.f__Veillonellaceae

p__Firmicutes.c_Bacilli.o__Gemellales.f__Gemellaceae

p__Firmicutes.c_Bacilli.o__Gemellales

p__Firmicutes.c_Bacilli.o__Gemellales.f__Gemellaceae.__

p__Proteobacteria.c__Betaproteobacteria.o__Neisseriales.f_Neisseriaceae

p__Proteobacteria.c__Betaproteobacteria.o__Neisseriales colon

colon

colon

colon

colon

colon

colon

stomach

stomach

stomach

stomach

stomach

stomach

stomach

stomach

stomach

stomach

stomach

stomach

stomach

stomach

stomach

stomach

stomach

stomach

stomach
3.6503

3.6503

3.6481

3.6393

3.6107

3.6099

3.6099

5.4670

5.4632

5.2180

5.2179

5.1109

5.1092

4.9740

4.8884

4.8013

4.7553

4.6845

4.6254

4.6225

4.5965

4.2689

4.2689

4.2676

4.2652

4.2471
0.0033

0.0033

0.0331

0.0163

0.0163

0.0331

0.0331

0.0003

0.0003

0.0012

0.0012

0.0074

0.0074

0.0074

0.0008

0.0003

0.0012

0.0035

0.0023

0.0023

0.0475

0.0010

0.0010

0.0034

0.0320

0.0320 
p__Fusobacteria.c__Fusobacteriia.o__Fusobacteriales.f_Leptotrichiaceae.g_Leptotrichia

stomach

4.2449

0.0109

__Firmicutes.c_Bacilli.o__Lactobacillales.f

stomach

4.2049

0.0109

p__Firmicutes.c_Bacilli.o__Lactobacillales.f__Carnobacteriaceae.g_Granulicatella

stomach

4.2049

0.0109

P_Fusobacteria.c__Fusobacteriia

stomach

4.2045

stomach

4.2023

p_Fusobacteria.c_Fusobacteriia.o__Fusobacteriales

stomach

4.2012

0.0345

p__Fusobacteria

stomach

4.1920

0.0345

__Fusobacteria.c_Fusobacteriia.o__Fusobacteriales.f__Leptotrichiaceae 
Supplementary Table 4. KO pathways identified by LEfSe analysis different between stomach and colonic microbiota(log LDA score $>2.0, p<0.05)$

\begin{tabular}{|c|c|c|c|c|c|c|}
\hline KO pathway & Level 3 & Gut site & $\begin{array}{l}\log _{10}(\text { LDA } \\
\text { score) }\end{array}$ & $p$-value & Level1 & Level 2 \\
\hline ko01051 & $\begin{array}{l}\text { Biosynthesis of } \\
\text { ansamycins }\end{array}$ & colon & 3.917 & 0.001 & Metabolism & $\begin{array}{l}\text { Metabolism of } \\
\text { terpenoids and } \\
\text { polyketides }\end{array}$ \\
\hline ko02030 & $\begin{array}{l}\text { Bacterial } \\
\text { chemotaxis }\end{array}$ & colon & 3.604 & 0.008 & $\begin{array}{l}\text { Cellular } \\
\text { Processes }\end{array}$ & Cell motility \\
\hline & & & & & $\begin{array}{l}\text { Genetic } \\
\text { Information }\end{array}$ & \\
\hline ko03020 & RNA polymerase & colon & 3.561 & 0.000 & Processing & Transcription \\
\hline ko00521 & $\begin{array}{l}\text { Streptomycin } \\
\text { biosynthesis }\end{array}$ & colon & 3.479 & 0.000 & Metabolism & $\begin{array}{l}\text { Biosynthesis of } \\
\text { other } \\
\text { secondary } \\
\text { metabolites }\end{array}$ \\
\hline ko00121 & $\begin{array}{l}\text { Secondary bile acid } \\
\text { biosynthesis }\end{array}$ & colon & 3.461 & 0.000 & Metabolism & $\begin{array}{l}\text { Lipid } \\
\text { metabolism }\end{array}$ \\
\hline ko02040 & $\begin{array}{l}\text { Flagellar assembly } \\
\text { Alanine, aspartate }\end{array}$ & colon & 3.433 & 0.026 & $\begin{array}{l}\text { Cellular } \\
\text { Processes }\end{array}$ & Cell motility \\
\hline ko00250 & $\begin{array}{l}\text { and glutamate } \\
\text { metabolism }\end{array}$ & colon & 3.380 & 0.000 & Metabolism & $\begin{array}{l}\text { Amino acid } \\
\text { metabolism }\end{array}$ \\
\hline
\end{tabular}




\begin{tabular}{|c|c|c|c|c|c|c|}
\hline ko00730 & $\begin{array}{l}\text { Thiamine } \\
\text { metabolism }\end{array}$ & colon & 3.339 & 0.000 & Metabolism & $\begin{array}{l}\text { Metabolism of } \\
\text { cofactors and } \\
\text { vitamins }\end{array}$ \\
\hline ko01055 & $\begin{array}{l}\text { Biosynthesis of } \\
\text { vancomycin group } \\
\text { antibiotics }\end{array}$ & colon & 3.333 & 0.001 & Metabolism & $\begin{array}{l}\text { Metabolism of } \\
\text { terpenoids and } \\
\text { polyketides }\end{array}$ \\
\hline ko00300 & Lysine biosynthesis & colon & 3.238 & 0.000 & Metabolism & $\begin{array}{l}\text { Amino acid } \\
\text { metabolism }\end{array}$ \\
\hline ko00511 & $\begin{array}{l}\text { Other glycan } \\
\text { degradation }\end{array}$ & colon & 3.212 & 0.001 & Metabolism & $\begin{array}{l}\text { Glycan } \\
\text { biosynthesis } \\
\text { and metabolism }\end{array}$ \\
\hline ko00900 & $\begin{array}{l}\text { Terpenoid } \\
\text { backbone } \\
\text { biosynthesis }\end{array}$ & colon & 3.122 & 0.005 & Metabolism & $\begin{array}{l}\text { Metabolism of } \\
\text { terpenoids and } \\
\text { polyketides }\end{array}$ \\
\hline ko00340 & $\begin{array}{l}\text { Histidine } \\
\text { metabolism }\end{array}$ & colon & 3.091 & 0.000 & Metabolism & $\begin{array}{l}\text { Amino acid } \\
\text { metabolism }\end{array}$ \\
\hline ko00625 & $\begin{array}{l}\text { Chloroalkane and } \\
\text { chloroalkene } \\
\text { degradation }\end{array}$ & colon & 3.064 & 0.033 & Metabolism & $\begin{array}{l}\text { Xenobiotics } \\
\text { biodegradation } \\
\text { and metabolism }\end{array}$ \\
\hline ko00770 & $\begin{array}{l}\text { Pantothenate and } \\
\text { CoA biosynthesis }\end{array}$ & colon & 3.046 & 0.008 & Metabolism & $\begin{array}{l}\text { Metabolism of } \\
\text { cofactors and } \\
\text { vitamins }\end{array}$ \\
\hline
\end{tabular}




\begin{tabular}{|c|c|c|c|c|c|c|}
\hline ko00312 & $\begin{array}{l}\text { beta-Lactam } \\
\text { resistance }\end{array}$ & colon & 3.044 & 0.000 & $\begin{array}{l}\text { Human } \\
\text { Diseases }\end{array}$ & $\begin{array}{l}\text { Drug } \\
\text { resistance: } \\
\text { antimicrobial }\end{array}$ \\
\hline ko00550 & $\begin{array}{l}\text { Peptidoglycan } \\
\text { biosynthesis }\end{array}$ & colon & 2.977 & 0.005 & Metabolism & $\begin{array}{l}\text { Glycan } \\
\text { biosynthesis } \\
\text { and metabolism }\end{array}$ \\
\hline ko00500 & $\begin{array}{l}\text { Starch and sucrose } \\
\text { metabolism }\end{array}$ & colon & 2.905 & 0.048 & Metabolism & $\begin{array}{l}\text { Carbohydrate } \\
\text { metabolism }\end{array}$ \\
\hline ko03420 & $\begin{array}{l}\text { Nucleotide excision } \\
\text { repair }\end{array}$ & colon & 2.903 & 0.001 & $\begin{array}{l}\text { Genetic } \\
\text { Information } \\
\text { Processing }\end{array}$ & $\begin{array}{l}\text { Replication and } \\
\text { repair }\end{array}$ \\
\hline ko00052 & $\begin{array}{l}\text { Galactose } \\
\text { metabolism }\end{array}$ & colon & 2.861 & 0.006 & Metabolism & $\begin{array}{l}\text { Carbohydrate } \\
\text { metabolism }\end{array}$ \\
\hline ko00908 & Zeatin biosynthesis & colon & 2.838 & 0.005 & Metabolism & $\begin{array}{l}\text { Metabolism of } \\
\text { terpenoids and } \\
\text { polyketides }\end{array}$ \\
\hline ko00030 & $\begin{array}{l}\text { Pentose phosphate } \\
\text { pathway }\end{array}$ & colon & 2.752 & 0.021 & Metabolism & $\begin{array}{l}\text { Carbohydrate } \\
\text { metabolism }\end{array}$ \\
\hline ko00760 & $\begin{array}{l}\text { Nicotinate and } \\
\text { nicotinamide } \\
\text { metabolism } \\
\text { Cysteine and } \\
\text { methionine } \\
\text { metabolism }\end{array}$ & colon & 2.722 & 0.003 & Metabolism & $\begin{array}{l}\text { Metabolism of } \\
\text { cofactors and } \\
\text { vitamins } \\
\text { Amino acid } \\
\text { metabolism }\end{array}$ \\
\hline
\end{tabular}




\begin{tabular}{|c|c|c|c|c|c|c|}
\hline ko00120 & $\begin{array}{l}\text { Primary bile acid } \\
\text { biosynthesis }\end{array}$ & colon & 2.703 & 0.000 & Metabolism & $\begin{array}{l}\text { Lipid } \\
\text { metabolism }\end{array}$ \\
\hline ko00531 & $\begin{array}{l}\text { Glycosaminoglycan } \\
\text { degradation }\end{array}$ & colon & 2.681 & 0.013 & Metabolism & $\begin{array}{l}\text { Glycan } \\
\text { biosynthesis } \\
\text { and metabolism }\end{array}$ \\
\hline ko04141 & $\begin{array}{l}\text { Protein processing } \\
\text { in endoplasmic } \\
\text { reticulum }\end{array}$ & colon & 2.605 & 0.000 & $\begin{array}{l}\text { Genetic } \\
\text { Information } \\
\text { Processing }\end{array}$ & $\begin{array}{l}\text { Folding, sorting } \\
\text { and } \\
\text { degradation }\end{array}$ \\
\hline ko00791 & $\begin{array}{l}\text { Atrazine } \\
\text { degradation }\end{array}$ & colon & 2.589 & 0.008 & Metabolism & $\begin{array}{l}\text { Xenobiotics } \\
\text { biodegradation } \\
\text { and metabolism }\end{array}$ \\
\hline ko00830 & Retinol metabolism & colon & 2.477 & 0.033 & Metabolism & $\begin{array}{l}\text { Metabolism of } \\
\text { cofactors and } \\
\text { vitamins }\end{array}$ \\
\hline ko04626 & $\begin{array}{l}\text { Plant-pathogen } \\
\text { interaction }\end{array}$ & colon & 2.460 & 0.008 & $\begin{array}{l}\text { Organismal } \\
\text { Systems }\end{array}$ & $\begin{array}{l}\text { Environmental } \\
\text { adaptation }\end{array}$ \\
\hline ko03450 & $\begin{array}{l}\text { Non-homologous } \\
\text { end-joining }\end{array}$ & colon & 2.436 & 0.001 & $\begin{array}{l}\text { Genetic } \\
\text { Information } \\
\text { Processing }\end{array}$ & $\begin{array}{l}\text { Replication and } \\
\text { repair }\end{array}$ \\
\hline ko04974 & $\begin{array}{l}\text { Protein digestion } \\
\text { and absorption }\end{array}$ & colon & 2.390 & 0.001 & $\begin{array}{l}\text { Organismal } \\
\text { Systems }\end{array}$ & $\begin{array}{l}\text { Digestive } \\
\text { system }\end{array}$ \\
\hline ko05146 & Amoebiasis & colon & 2.365 & 0.004 & $\begin{array}{l}\text { Human } \\
\text { Diseases }\end{array}$ & $\begin{array}{l}\text { Infectious } \\
\text { disease: } \\
\text { parasitic }\end{array}$ \\
\hline
\end{tabular}




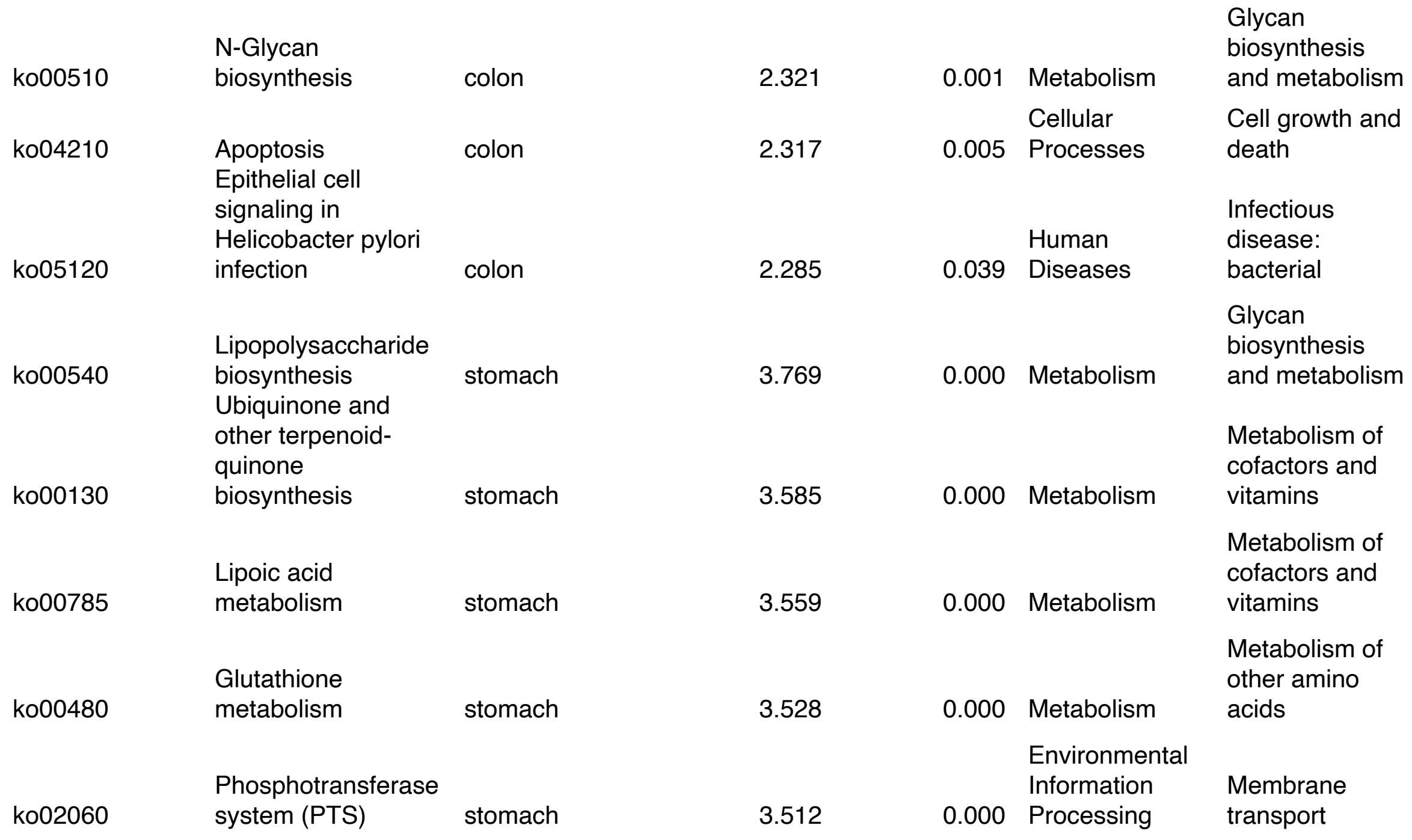




\begin{tabular}{|c|c|c|c|c|c|c|}
\hline ko00790 & Folate biosynthesis & stomach & 3.462 & 0.000 & Metabolism & vitamins \\
\hline ko00780 & Biotin metabolism & stomach & 3.449 & 0.000 & Metabolism & $\begin{array}{l}\text { Metabolism of } \\
\text { cofactors and } \\
\text { vitamins }\end{array}$ \\
\hline ko02010 & $\mathrm{ABC}$ transporters & stomach & 3.292 & 0.000 & $\begin{array}{l}\text { Environmental } \\
\text { Information } \\
\text { Processing }\end{array}$ & $\begin{array}{l}\text { Membrane } \\
\text { transport }\end{array}$ \\
\hline ko00660 & $\begin{array}{l}\text { Ascorbate and } \\
\text { aldarate metabolism } \\
\text { C5-Branched } \\
\text { dibasic acid } \\
\text { metabolism }\end{array}$ & stomach & 3.221 & 0.002 & Metabolism & $\begin{array}{l}\text { Carbohydrate } \\
\text { metabolism } \\
\text { Carbohydrate } \\
\text { metabolism }\end{array}$ \\
\hline$?$ & & h & 2ח & ค० & $\begin{array}{l}\text { Genetic } \\
\text { Information }\end{array}$ & Folding, sorting \\
\hline ko00910 & $\begin{array}{l}\text { Nitrogen } \\
\text { metabolism }\end{array}$ & stomach & 3.208 & 0.000 & Metabolism & $\begin{array}{l}\text { Energy } \\
\text { metabolism }\end{array}$ \\
\hline ko00020 & $\begin{array}{l}\text { Citrate cycle (TCA } \\
\text { cycle) }\end{array}$ & stomach & 3.137 & 0.000 & Metabolism & $\begin{array}{l}\text { Carbohydrate } \\
\text { metabolism }\end{array}$ \\
\hline ko00920 & Sulfur metabolism & stomach & 3.132 & 0.000 & Metabolism & $\begin{array}{l}\text { Energy } \\
\text { metabolism }\end{array}$ \\
\hline
\end{tabular}

Metabolism of cofactors and

Metabolism of cofactors and

Environmental Information

Membrane transport

Carbohydrate

Carbohydrate metabolism

and

degradation

Energy

Carbohydrate

Energy 


\begin{tabular}{|c|c|c|c|c|c|c|}
\hline ko00450 & $\begin{array}{l}\text { Selenocompound } \\
\text { metabolism }\end{array}$ & stomach & 3.120 & 0.000 & Metabolism & $\begin{array}{l}\text { Metabolism of } \\
\text { other amino } \\
\text { acids }\end{array}$ \\
\hline ko00473 & $\begin{array}{l}\text { D-Alanine } \\
\text { metabolism }\end{array}$ & stomach & 3.108 & 0.002 & Metabolism & $\begin{array}{l}\text { Metabolism of } \\
\text { other amino } \\
\text { acids }\end{array}$ \\
\hline ko00650 & $\begin{array}{l}\text { Butanoate } \\
\text { metabolism }\end{array}$ & stomach & 3.003 & 0.000 & Metabolism & $\begin{array}{l}\text { Carbohydrate } \\
\text { metabolism }\end{array}$ \\
\hline ko00564 & $\begin{array}{l}\text { Glycerophospholipid } \\
\text { metabolism } \\
\text { Glyoxylate and }\end{array}$ & stomach & 2.982 & 0.000 & Metabolism & $\begin{array}{l}\text { Lipid } \\
\text { metabolism }\end{array}$ \\
\hline ko00630 & $\begin{array}{l}\text { dicarboxylate } \\
\text { metabolism }\end{array}$ & stomach & 2.960 & 0.021 & Metabolism & $\begin{array}{l}\text { Carbohydrate } \\
\text { metabolism }\end{array}$ \\
\hline ko05322 & $\begin{array}{l}\text { Systemic lupus } \\
\text { erythematosus } \\
\text { Biosvnthesis of }\end{array}$ & stomach & 2.948 & 0.016 & $\begin{array}{l}\text { Human } \\
\text { Diseases }\end{array}$ & $\begin{array}{l}\text { Immune } \\
\text { disease }\end{array}$ \\
\hline ko01053 & $\begin{array}{l}\text { siderophore group } \\
\text { nonribosomal } \\
\text { peptides }\end{array}$ & stomach & 2.942 & 0.037 & Metabolism & $\begin{array}{l}\text { Metabolism of } \\
\text { terpenoids and } \\
\text { polyketides }\end{array}$ \\
\hline ko00620 & $\begin{array}{l}\text { Pyruvate } \\
\text { metabolism }\end{array}$ & stomach & 2.905 & 0.003 & Metabolism & $\begin{array}{l}\text { Carbohydrate } \\
\text { metabolism }\end{array}$ \\
\hline ko00633 & $\begin{array}{l}\text { Nitrotoluene } \\
\text { degradation }\end{array}$ & stomach & 2.890 & 0.010 & Metabolism & $\begin{array}{l}\text { Xenobiotics } \\
\text { biodegradation } \\
\text { and metabolism }\end{array}$ \\
\hline ko00010 & $\begin{array}{l}\text { Glycolysis / } \\
\text { Gluconeogenesis }\end{array}$ & stomach & 2.880 & 0.001 & Metabolism & $\begin{array}{l}\text { Carbohydrate } \\
\text { metabolism }\end{array}$ \\
\hline
\end{tabular}




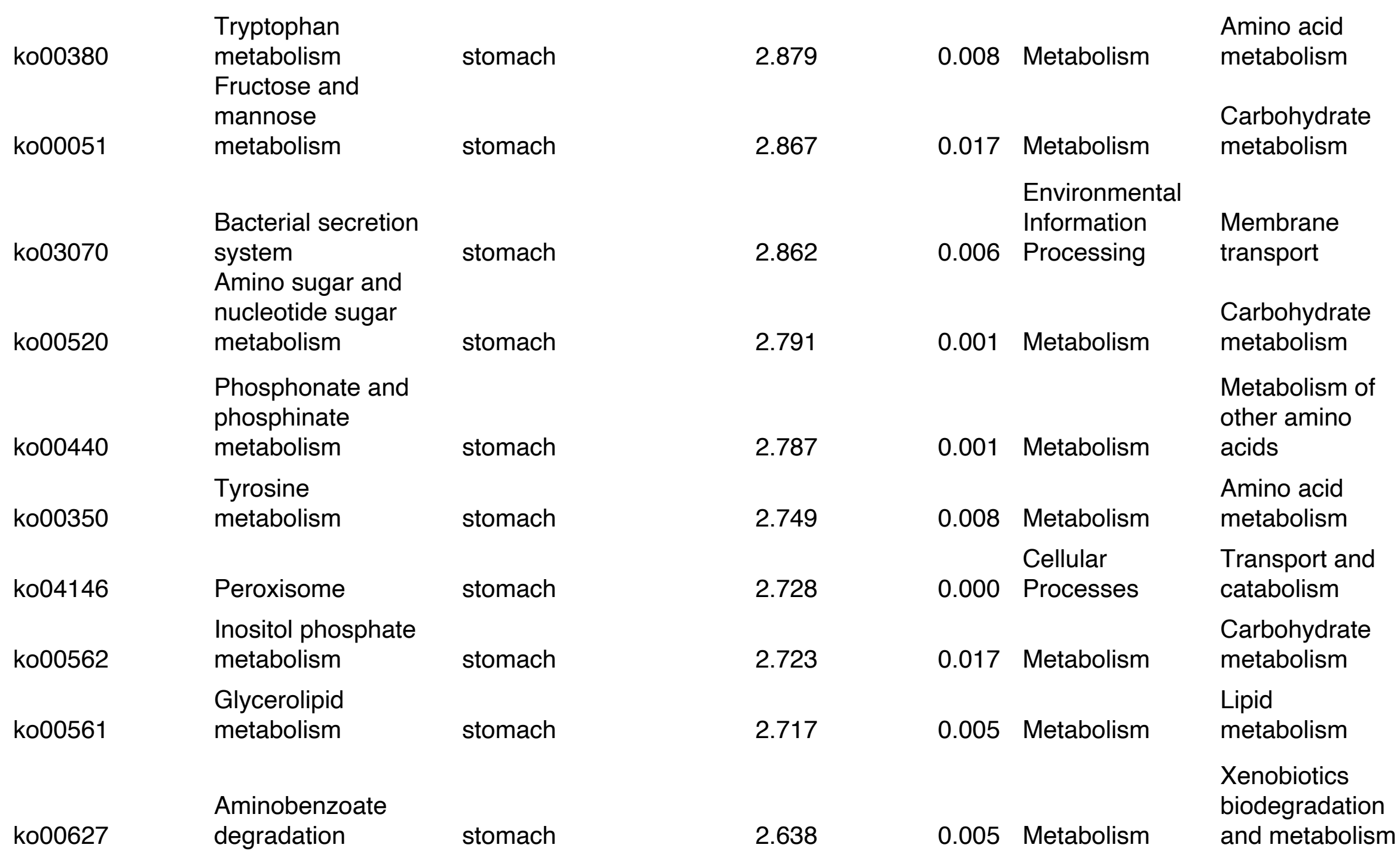




\begin{tabular}{|c|c|c|c|c|c|c|}
\hline ko00196 & $\begin{array}{l}\text { Photosynthesis - } \\
\text { antenna proteins }\end{array}$ & stomach & 2.628 & 0.028 & Metabolism & $\begin{array}{l}\text { Energy } \\
\text { metabolism }\end{array}$ \\
\hline ko00361 & $\begin{array}{l}\text { Chlorocyclohexane } \\
\text { and chlorobenzene } \\
\text { degradation }\end{array}$ & stomach & 2.609 & 0.013 & Metabolism & $\begin{array}{l}\text { Xenobiotics } \\
\text { biodegradation } \\
\text { and metabolism }\end{array}$ \\
\hline ko03008 & $\begin{array}{l}\text { Ribosome } \\
\text { biogenesis in } \\
\text { eukaryotes }\end{array}$ & stomach & 2.486 & 0.000 & $\begin{array}{l}\text { Genetic } \\
\text { Information } \\
\text { Processing }\end{array}$ & Translation \\
\hline ko05150 & $\begin{array}{l}\text { Staphylococcus } \\
\text { aureus infection }\end{array}$ & stomach & 2.378 & 0.001 & $\begin{array}{l}\text { Human } \\
\text { Diseases }\end{array}$ & $\begin{array}{l}\text { Infectious } \\
\text { disease: } \\
\text { bacterial }\end{array}$ \\
\hline ko05142 & $\begin{array}{l}\text { Chagas disease } \\
\text { (American } \\
\text { trypanosomiasis) }\end{array}$ & stomach & 2.096 & 0.001 & $\begin{array}{l}\text { Human } \\
\text { Diseases }\end{array}$ & $\begin{array}{l}\text { Infectious } \\
\text { disease: } \\
\text { parasitic }\end{array}$ \\
\hline
\end{tabular}

